\title{
Numerical Taxonomy of Vibrios Isolated from Estuarine Environments
}

\author{
J. B. KAPER, $\dagger$ H. LOCKMAN, $\dagger$ E. F. REMMERS, K. KRISTENSEN, $\ddagger$ AND R. R. COLWELL* \\ Department of Microbiology, University of Maryland, College Park, Maryland 20742
}

\begin{abstract}
We used numerical taxonomy procedures to analyze data obtained from 227 strains belonging to either the genus Vibrio or related genera; included were pathogenic and nonpathogenic vibrios isolated from a variety of samples and geographic locations. Each strain was tested for 150 unit characters. At a similarity coefficient of 70 to $75 \%$ or more, the 227 strains clustered into 33 phena, representing Vibrio anguillarum, Vibrio cholerae, Vibrio parahaemolyticus, Vibrio metschnikovii, Aeromonas hydrophila, and new species of Vibrio. Non-O1 and O1 serovars of $V$. cholerae clustered at the species level of relationship (i.e., at a similarity coefficient of $\geq 75 \%$ ). Furthermore, subdivision of $V$. cholerae into classical, El Tor, proteus, and albensis biovars was not observed. In fact, the biovar proteus. was found to warrant separate species status as Vibrio proteus. Only the sucrose-negative members of Heiberg group $V$ were distinguishable as a separate cluster and were recognized as a separate biovar. Sucrose-positive strains and urease-positive strains of $V$. parahaemolyticus were identified. The differences among the four groups of $V$. anguillarum examined did not warrant recognition of biovars.
\end{abstract}

Species of the genus Vibrio have become increasingly important, especially in medical and environmental microbiology. The type species of Vibrio, Vibrio cholerae, is the causative agent of Asiatic cholera, and Vibrio parahaemolyticus is associated with food poisoning, especially from ingestion of contaminated seafood. Vibrios have been shown to be important in the aquatic environment, for they constitute a significant portion of marine and estuarine bacterial populations in water and are associated with aquatic animals and plants. Species of Vibrio found in the environment include pathogens for fish (20), snails (24), and other invertebrates.

The taxonomy of the genus Vibrio has undergone major revision in recent years. In Bergey's Manual of Determinative Bacteriology, 7 th ed (10), Breed et al. listed 34 species for the genus and included aerobes, facultative anaerobes, and obligate anaerobes, strains of which had little in common except for being curved, motile rods. In the 8th edition of Bergey's Manual (82), Shewan and Veron took into account more recent knowledge and provided a much improved classification, but they still left many unanswered questions.

Recently several new species have been pro-

† Present address: Center for Vaccine Development, University of Maryland Medical School, Baltimore, MD 21201.

$\ddagger$ Present address: Veterinary Faculty for F.A.O. Fellows \& Scholars, 13 Bulowsvej, DK 1870 Copenhagen V, Denmark. posed for inclusion in the genus Vibrio. Lee et al. $(60,62)$ redescribed Vibrio metschnikovii and also named a new species, Vibrio fluvialis (the group F vibrios; i.e., group EF6). The latter is considered to be intermediate between the genera Vibrio and Aeromonas (62). An organism capable of extensive tissue damage or death or both in humans was demonstrated by Clark and Steigerwalt (12) to be closely related to species of the genus Vibrio and was subsequently named Vibrio vulnificus by Farmer (28). These organisms were formerly referred to as "lactosepositive vibrios." Vibrio damsela (65) and Vibrio hollisae (42) have also been described. There is no doubt that other new species will be proposed as current research continues.

The objective of this study was to examine a large set of vibrios representing as many species as could be obtained from a variety of sources, including environmental and medical, throughout the world. Numerical taxonomy was used to measure phenotypic similarities and differences and to analyze the relationships, if any, among phenotypes associated with geographic sources or types of sample. Our working hypothesis was that answers to environmental and medical questions concerning vibrios can be best answered by using an integrated (i.e., polyphasic) approach to the systematics of the genus Vibrio (13). Thus, the results obtained in this study are discussed in light of other criteria, as well as numerical taxonomy. 
TABLE 1. Sources of strains included in the numerical taxonomy analysis

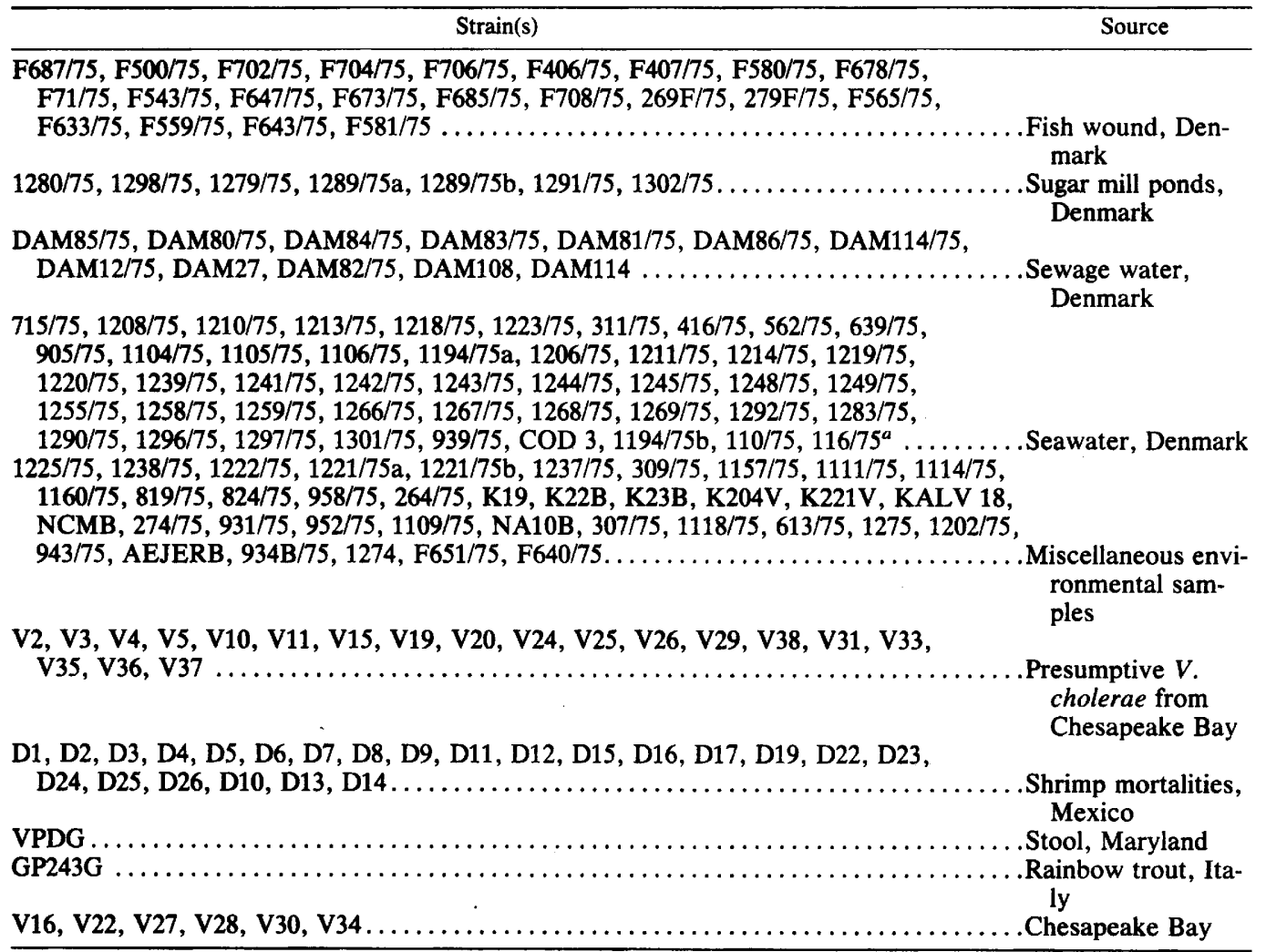

${ }^{a}$ The culture received as strain $1290 / 75$ yielded two distinct colonial types, both of which were included in the analyses.

\section{MATERIALS AND METHODS}

Bacterial strains. A total of 227 strains was examined by numerical taxonomy methods. These strains included pathogenic and nonpathogenic vibrios and vibrio-like bacteria isolated from the Chesapeake Bay, Denmark, and Mexico and represented strains isolated from a variety of sources, including fish, shrimp, seawater, and sewage. The clinical isolates included strains of $V$. cholerae isolated from various sources throughout the world. Reference cultures, which represented a diverse range of facultative anaerobes, were obtained from the American Type Culture Collection. The strains used in this study and their sources are shown in Tables 1 and 2.

Upon isolation or receipt from a collection, each culture was examined for purity by serial streaking three times on tryptic soy agar (TSA; Difco Laboratories, Detroit, Mich.). Stock cultures were maintained on TSA slants that were overlaid with sterile mineral oil and stored at $25^{\circ} \mathrm{C}$.

Characterization of strains. Each strain was examined for 150 unit characters. All inocula were taken from cultures that were less than $48 \mathrm{~h}$ old. Whenever possible, TSA was used as the basal medium for tests involving dyes, salt, etc. Unless otherwise stated, all inoculated media were incubated at $25^{\circ} \mathrm{C}$, and all media contained $\mathrm{NaCl}$ at a final concentration of $0.5 \%$. Tests were repeated in the event of inconclusive results.

Morphology. Colonial morphologies were determined on TSA after incubation for 2 days by using the criteria of Colwell and Weibe (17). The following characteristics were scored for each strain: the presence of creamy white, yellow, orange or red, or diffusible pigments; translucent, opaque, shiny or matte, round, flat, raised, domed, wrinkled, or spreading colonies; and undulate, irregular, or rhizoid colony edges. The presence of fluorescein and the presence of pyocyanin were detected by examining with a fluorescent light the growth obtained after 7 or more days of incubation on the media described by King et al. (56).

Micromorphology and Gram-staining reactions were recorded from heat-fixed smears of fresh cultures after staining with the Hucker modification of the Gram stain (45). We also recorded the presence of pleomorphism, curved and straight rods, chains, filaments, and granular cytoplasm. The approximate ratio of rod length to rod width was measured, and motility was determined both microscopically (by wet mount) and macroscopically (SIM medium [Difco]).

Physiology and resistance to physical and chemical factors. The ability to grow at 5, 10, 15, 37, 42, and $44^{\circ} \mathrm{C}$ was determined by using TSA and air incubators. 
TABLE 2. Reference strains included in the numerical taxonomy analyses

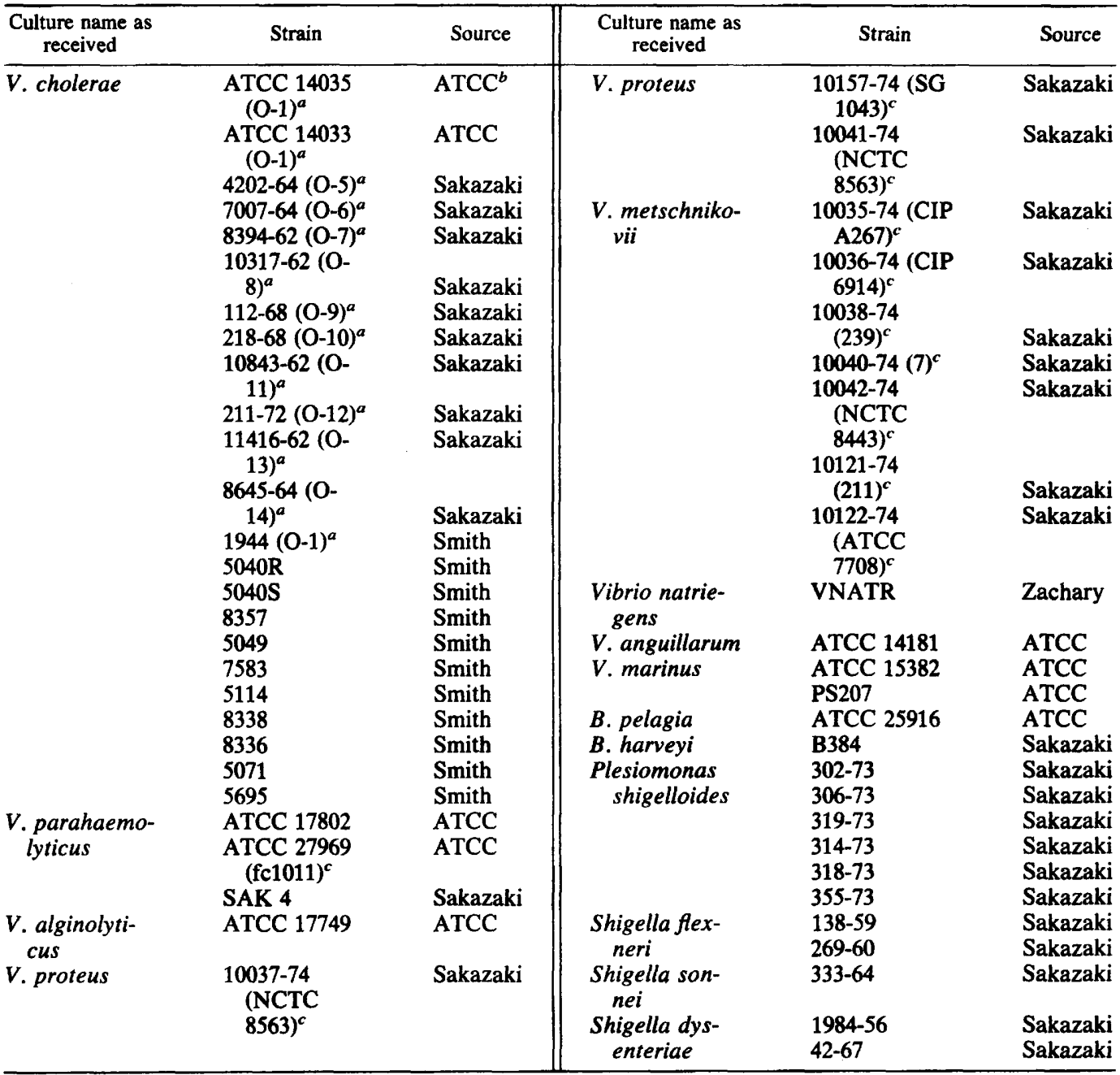

a The information in parentheses is the serovar of the strain according to the scheme of $\mathbf{R}$. Sakazaki (personal communication).

${ }^{b}$ ATCC, American Type Culture Collection, Rockville, Md.

$c$ The designations in parentheses are other strain designations.

The ability to grow at pH 4.5, 8.0, 9.0, and 9.5 was determined by using TSA adjusted to the appropriate $\mathrm{pH}$ with $\mathrm{HCl}$ or $\mathrm{NaOH}$. The ability to grow in final $\mathrm{NaCl}$ concentrations of $0,3,7$, and $10 \%$ was determined by using $1 \%$ peptone broth supplemented with appropriate amounts of $\mathrm{NaCl}$.

The ability to tolerate brilliant green $(0.00125 \%)$, pyronin $\mathrm{G}(0.001 \%)$, neutral red $(0.001 \%)$, and crystal violet $(0.001 \%)$ was tested on TSA appropriately modified. Cultures were also tested for the ability to grow on thiosulfate-citrate-bile salts (TCBS) agar (Difco) and salmonella-shigella (SS) agar (Difco). Susceptibility to the following antibiotics was assessed by the disk diffusion method (BBL Microbiology Systems, Cockeysville, Md.): gentamicin $(10 \mu \mathrm{g})$, penicillin (10 $\mathrm{U})$, streptomycin $(10 \mu \mathrm{g})$, chloramphenicol $(30 \mu \mathrm{g})$, erythromycin $(15 \mu \mathrm{g})$, kanamycin $(30 \mu \mathrm{g})$, chlortetracycline $(30 \mu \mathrm{g})$, novobiocin $(30 \mu \mathrm{g})$, polymyxin B (50 $U)$, oxytetracycline $(30 \mu \mathrm{g})$, nalidixic acid $(30 \mu \mathrm{g})$, tetracycline $(30 \mu \mathrm{g})$, and ampicillin $(10 \mu \mathrm{g})$. Susceptibility to vibriostatic agent 0/129 (2,4-diamino-6,7-diisopropylpteridine) was determined by dropping a crystal of 2,4-diamino-6,7 diisopropylpteridine phosphate (Sigma Chemical Co., St. Louis, Mo.) onto a TSA plate immediately after seeding, as for antibiotic testing. Susceptibility to vibriostatic agent $0 / 129$ was recorded as positive when a zone of clearing was observed. Strains which gave doubtful or unusual reactions were further tested for minimal inhibitory concentrations of vibriostatic agent $0 / 129$ by using plates of heart infusion agar (Difco) containing $0,5,10$, $50,100,150$, or $300 \mu \mathrm{g}$ of 2,4 -diamino-6,7-diisopro- 


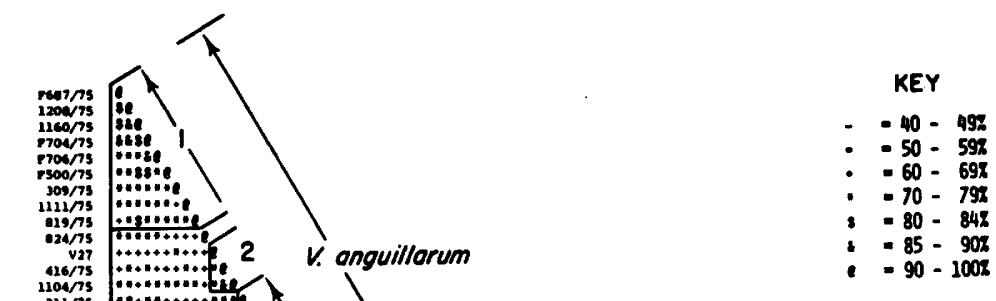




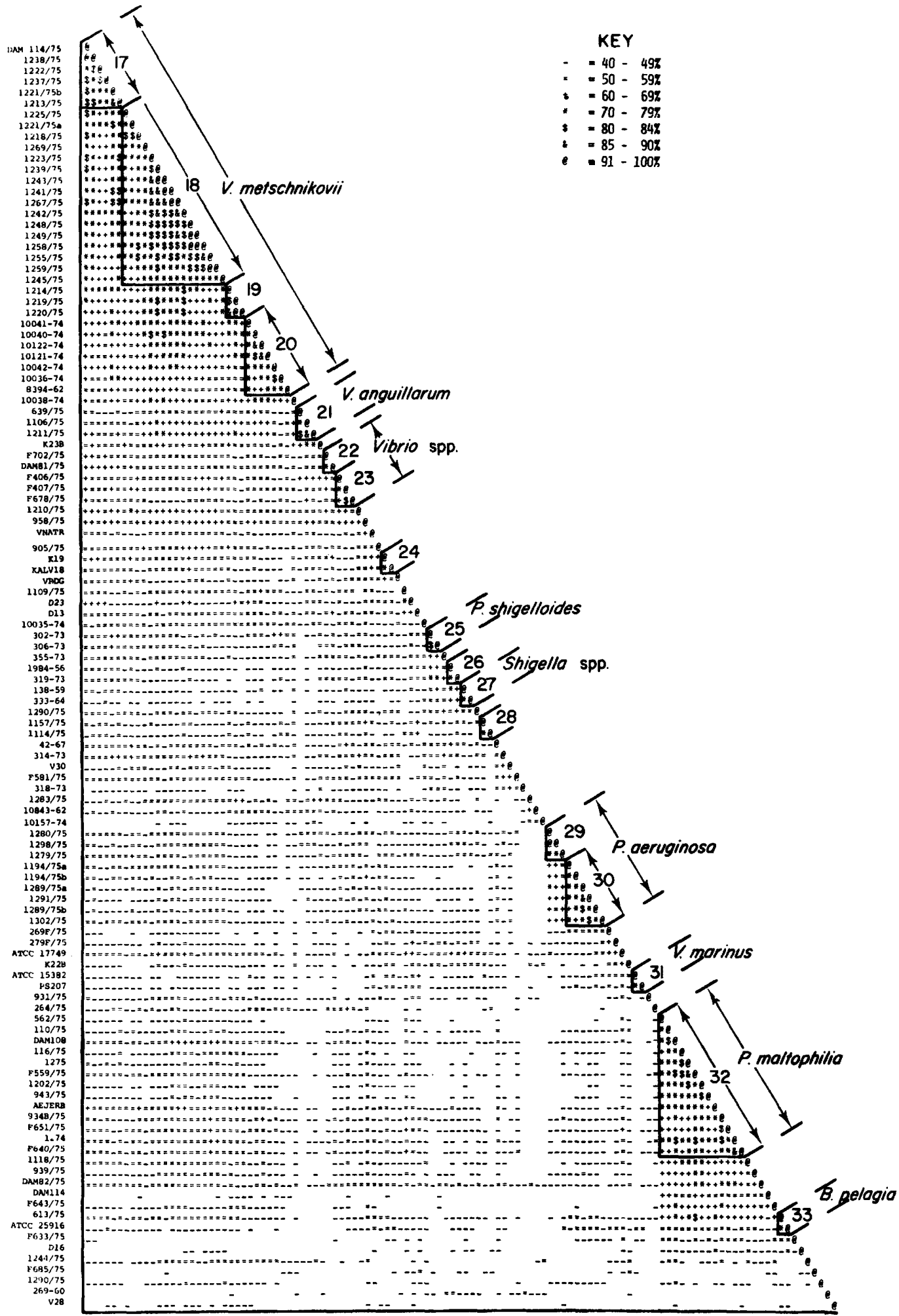

FIG. 2. Similarity matrix for phena 17 through 33 , determined by using the Jaccard similarity coefficient and unweighted average linkage. 


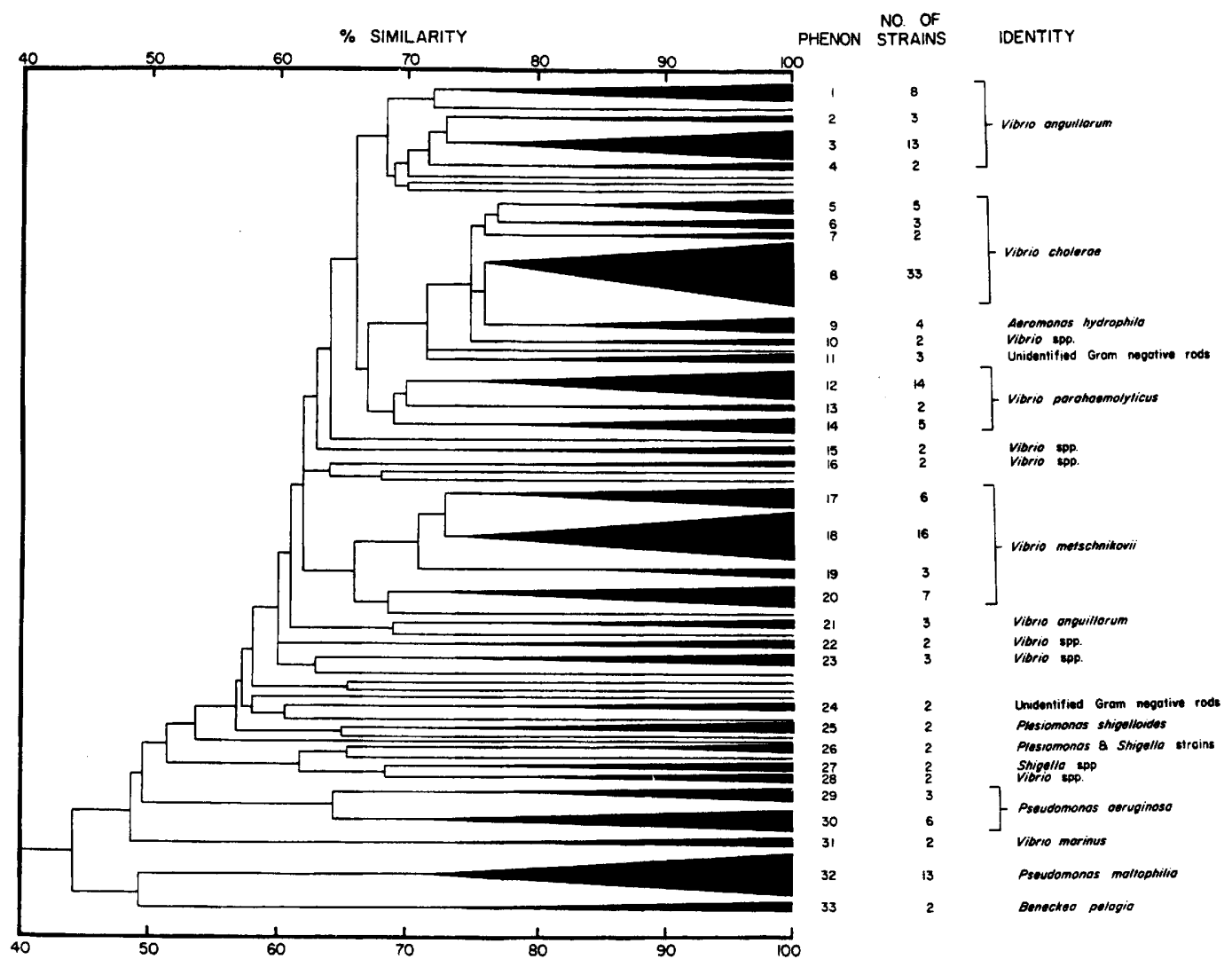

FIG. 3. Simplified dendrogram of strains examined in this study, based on the Jaccard similarity coefficient and unweighted average linkage.

pylpteridine phosphate (Sigma) per ml (J. V. Lee, P. A. Court, T. J. Donovan, and A. L. Furniss, J. Appl. Bacteriol. 45:viii, 1978).

Biochemical tests. The following tests were read after incubation for 5 days unless otherwise noted: arginine dihydrolase, lysine decarboxylase, ornithine decarboxylase, and histidine decarboxylase (Moeller medium [Difco]) (67); nitrate and nitrite reduction, methyl red, Voges-Proskauer, and phenylalanine deaminase (prepared culture medium [Difco]); oxidase (58); catalase (17); hydrogen sulfide production from sodium thiosulfate (triple sugar iron agar [TSI; Difco]) after 7 days; urease production (broth medium of Stuart et al. [95]); indole production (1\% peptone broth and Kovacs reagent [57]); growth and acid production in iodoacetate after incubation for 7 days (method of Colwell and Wiebe [17]); levan production after incubation for 7 days on TSA supplemented with $5 \%$ (wt/vol) sucrose; and ammonia production (17).

The test for fermentative or oxidative metabolism of glucose was performed by the method of Hugh and Leifson (47); the test media were examined after incubation for 1, 2, and 7 days. Acid production from each of the following carbohydrates was determined by using the medium of Hugh and Leifson (Difco) supplemented with the carbohydrate at a final concentration of $1 \%$ (wt/vol): adonitol, amygdalin, arabinose, butanol, cellobiose, dextrin, dulcitol, erythritol, etha- nol, fructose, galactose, glycerol, inositol, lactose, mannitol, mannose, melezitose, melibiose, raffinose, rhamnose, salicin, sorbitol, sorbose, sucrose, trehalose, and xylose. Carbohydrate solutions were filter sterilized and aseptically added to basal medium which had been autoclaved. The resulting media were dispensed in 24-well cluster dishes (Bellco Glass, Inc., Vineland, N.J.) (90) and inoculated by using a multipoint inoculator (66). Because of the high ratio of surface area to volume of the cluster dish wells, initial acid reactions often became alkaline due to oxidation. To guard against this possibility, reactions were read and recorded after incubation for 1, 2, 5, and 7 days. Production of gas from glucose and lactose was tested for in the oxidative-fermentative (OF) basal medium of Hugh and Leifson without agar. Inverted Durham tubes were inserted into the broth to capture any gas produced by the culture. Formation of gas was recorded after incubation for 7 days.

Degradation of organic compounds. Unless specified otherwise, all of the tests were read after incubation for 7 and 14 days. Gelatin hydrolysis was tested by the method of Smith and Goodner (84). Hemolysis was tested on TSA basal medium supplemented with sheep erythrocytes at a final concentration of $5 \%(\mathrm{vol} / \mathrm{vol})$. Plates were examined after $48 \mathrm{~h}$.

Deoxyribonuclease was detected on DNase test agar (Difco) supplemented with toluidine blue at a final 
concentration of $0.1 \%(\mathrm{wt} / \mathrm{vol})$ (9). Plates were read after 3,5, and 7 days and then discarded.

The production of lipase was determined by the method of Sierra (83), using four different substrates (viz. Tween 20, Tween 40, Tween 60, and Tween 80) at final concentrations of $1 \%$ (wt/vol). Esculin hydrolysis was determined by the method of Sneath (87). The following two reactions were recorded: (i) browning of agar resulting from precipitation of ferric ion in the medium; and (ii) disappearance of the characteristic fluorescence under ultraviolet (UV) light. Degradation of allantoin and tyrosine was detected by the method of Gordon (35). Lecithinase was detected by the method of Baird-Parker (3); the medium úsed consisted of $100 \mathrm{ml}$ of egg yolk emulsion (Oxoid Ltd., London, England) and $900 \mathrm{ml}$ of TSA. Another method of Baird-Parker (3) was used to detect phosphatase activity; in this test, phenophthalein diphosphate was employed as the substrate. Cellulose hydrolysis was determined on Whatman filter paper partially immersed in the salt solution recommended by DuBos (23). Casein hydrolysis was determined on TSA containing 5\% skim milk.

Chitin digestion was detected by the method of $\mathrm{Hsu}$ and Lockwood (44), and chondroitinase activity was detected by the method of Smith and Willett (86).

Utilization of sole carbon sources. Sole carbon source utilization tests were performed by the method of Stevenson (94). The carbon sources used included $\beta$ alanine, DL-alanine, arginine, aspartate, glycine, inulin, lysine, phenylalanine, proline, propanol, serine, sodium acetate, sodium alginate, sodium citrate, sodium formate, sodium glutamate, sodium lactate, sodium malonate, sodium mucate, sodium pyruvate, sodium succinate, sodium tartrate, tryptophan, and uracil. The substrates serving as carbon sources were sterilized as $10 \%$ (wt/vol) solutions by tyndallization for $1 \mathrm{~h}$ at $100^{\circ} \mathrm{C}$ on 3 consecutive days. To obtain $500 \mathrm{ml}$ (final volume) of medium containing a sole carbon source, $440 \mathrm{ml}$ of water agar base containing $5 \mathrm{~g}$ of purified agar (Difco) and $2.5 \mathrm{~g}$ of $\mathrm{NaCl}$ was autoclaved at $121^{\circ} \mathrm{C}$ for $15 \mathrm{~min}$. A supporting nutrient base was made up of $80.4 \mathrm{~g}$ of yeast nitrogen base (Difco) and $0.12 \mathrm{~g}$ of Casamino Acids (Difco) in 1,200 ml of glass-distilled water. The solution was filter sterilized and then neutralized by adding $300 \mathrm{ml}$ of $\mathrm{K}_{2} \mathrm{HPO}_{4}(10 \%$, wt/vol) that had been autoclaved at $121^{\circ} \mathrm{C}$ for $15 \mathrm{~min}$. A $50-\mathrm{ml}$ portion of the buffered base was added aseptically to the water agar, which had been cooled to $30^{\circ} \mathrm{C}$, and then $10 \mathrm{ml}$ of a $10 \%$ solution of a carbon source was added. Each lot of medium was thoroughly mixed and dispensed via a peristaltic pump into polystyrene cluster dishes, which were subsequently inoculated by using a multipoint inoculator. The inoculated plates were examined after incubation for 14 days, and the presence or absence of growth was recorded after comparisons with positive controls (glucose as the carbon source) and negative controls (medium lacking the carbon source).

Computer analysis. The test results were coded 0 for negative results, 1 for positive results, and 9 for noncomparable or missing data. The combined data were entered into a Univac model 1108 computer and analyzed to determine whether any of the tests were uniformly positive or negative and, therefore, nondiscriminatory. Such tests and other tests believed to be highly subjective (viz., pleomorphism of cells) were deleted from the data matrix, leaving a total of 129 tests for the final analysis.

The data matrix was prepared by using the simple matching coefficient (92), which includes both positive and negative matches, and the Jaccard coefficient (88), which excludes negative matches. Clustering was by unweighted average linkage and by single linkage (89). The programs used included the Taxan 6 and IGPS3 program packages, both of which were available on the Univac model 1108 computer at the University of Maryland.

\section{RESULTS}

Vibrio spp. The 227 strains examined clustered into 33 phena at a similarity coefficient of 70 to $75 \%$ or more when the Jaccard coefficient was used and clustering was achieved by unweighted average linkage. The similarity matrix derived in this way is shown in Fig. 1 and 2, and the related dendrogram is shown in Fig. 3.

The first four clusters contained strains of Vibrio anguillarum. Phenon 1 consisted of eight environmental isolates from Denmark, including four strains isolated from diseased fish (Fig. 3). Phenon 2 was composed of two strains from Denmark and one strain, strain V27, isolated from the Chesapeake Bay. Cluster 3 consisted of 13 strains, 11 from fish and waters of Denmark, one (strain V34) from the Chesapeake Bay, and the reference (type) strain of the species, $V$. anguillarum ATCC 14181. Cluster 4 consisted of a pair of Danish isolates, strains NA10B and $307 / 75$.

The strains of all four of these clusters were very similar to $V$. anguillarum $(25,82)$. These strains were gram-negative, motile, facultatively anaerobic, rod-shaped organisms which are oxidase positive and susceptible to vibriostatic agent $0 / 129$ (Table 3). They grew in media containing $3 \% \mathrm{NaCl}$ but not in media lacking $\mathrm{NaCl}$ and produced acid, but no gas, from glucose, fructose, mannitol, sucrose, and trehalose. These strains were negative for acid production from adonitol, inositol, lactose, sorbose, and xylose and produced a variety of extracellular enzymes which degraded starch, gelatin, deoxyribonucleic acid (DNA), and Tween 20, Tween 40 , Tween 60 , and Tween 80 . They were able to utilize DL-alanine, citrate, inulin, proline, pyruvate, and serine as sole carbon sources, but not $\beta$-alanine, alginate, formate, lysine, malonate, tryptophan, or uracil. Most of the strains were positive for arginine dihydrolase but negative for lysine and ornithine decarboxylases.

Several phenotypic differences were observed among the clusters, as shown in Table 3. For example, strains in clusters 1 and 4 were able to grow at $42^{\circ} \mathrm{C}$; this characteristic was variable in the case of clusters 2 and 3 . Most of the $V$. anguillarum strains did not grow at this tempera- 
TABLE 3. Characteristics of phena 1 through $11^{a}$

\begin{tabular}{|c|c|c|c|c|c|c|c|c|c|c|c|}
\hline \multirow[b]{2}{*}{ Determination } & \multicolumn{11}{|c|}{$\%$ of strains positive in phenon: } \\
\hline & $\begin{array}{c}1 \\
(n=9)\end{array}$ & $\begin{array}{c}2 \\
(n=3)\end{array}$ & $\begin{array}{c}3 \\
(n=13)\end{array}$ & $\left(\begin{array}{c}4 \\
(n)\end{array}\right)$ & $\begin{array}{c}5 \\
(n=5)\end{array}$ & $\begin{array}{c}6 \\
(n=3)\end{array}$ & $\left(\begin{array}{c}7 \\
n=2)\end{array}\right.$ & $\left(\begin{array}{c}8 \\
(n=33)\end{array}\right.$ & $(n \stackrel{9}{=} 4)$ & $\begin{array}{c}10 \\
(n=2)\end{array}$ & $\begin{array}{c}11 \\
(n=3)\end{array}$ \\
\hline \multicolumn{12}{|l|}{$\begin{array}{l}\text { Colonial mor- } \\
\text { phology }\end{array}$} \\
\hline White or cream & $13(8)^{b}$ & 33 & 15 & 50 & 80 & 0 & 0 & $19(32)$ & 0 & 100 & 100 \\
\hline Yellow & $38(8)$ & 0 & 8 & 0 & 20 & 0 & 0 & $0(32)$ & 0 & 0 & 0 \\
\hline Colorless & 11 & 67 & 77 & 50 & $\mathbf{0}$ & 100 & 100 & 81(32) & 100 & 0 & $\mathbf{0}$ \\
\hline $\begin{array}{l}\text { Diffusible pig- } \\
\text { ment }\end{array}$ & $\mathbf{0}$ & 0 & $\mathbf{0}$ & $\mathbf{0}$ & $\mathbf{0}$ & 0 & $\mathbf{0}$ & 3 & 0 & $\mathbf{0}$ & $\mathbf{0}$ \\
\hline Mucoid & $\mathbf{0}$ & 0 & 0 & 0 & 0 & $\mathbf{0}$ & 0 & $0(32)$ & 0 & 0 & $\mathbf{0}$ \\
\hline Matt & 0 & $\mathbf{0}$ & 0 & 0 & 0 & 0 & 0 & 0 & 0 & 0 & 0 \\
\hline Translucent & 67 & 100 & 85 & 100(1) & $100(4)$ & 100 & 100 & 82 & 100 & 50 & 33 \\
\hline Round & 100 & 100 & 100 & 100 & 100 & 100 & 100 & 100 & 100 & 50 & 100 \\
\hline $\begin{array}{l}\text { Irregular mar- } \\
\text { gin }\end{array}$ & $\mathbf{0}$ & $\mathbf{0}$ & 0 & $\mathbf{0}$ & $\mathbf{0}$ & $\mathbf{0}$ & 0 & 0 & 0 & 50 & 0 \\
\hline Rhizoid margin & $\mathbf{0}$ & $\mathbf{0}$ & 0 & $\mathbf{0}$ & 0 & 0 & 0 & 0 & 0 & 0 & 0 \\
\hline Flat & 0 & 33 & 0 & 0 & $\mathbf{0}$ & 33 & 0 & 3 & 0 & 0 & $0(2)$ \\
\hline Spreading & $\mathbf{0}$ & $\mathbf{0}$ & 0 & 0 & $\mathbf{0}$ & 0 & $\mathbf{0}$ & $\mathbf{0}$ & 0 & 0 & 0 \\
\hline \multicolumn{12}{|l|}{ Micromorpholo- } \\
\hline $\begin{array}{l}\text { Gram variable } \\
\text { Length/width } \\
\text { ratio of } \leq 6: 1\end{array}$ & 0 & $0(2)$ & $0(12)$ & 0 & 200 & 0 & 0 & 0 & 0 & 0 & 0 \\
\hline Filaments & $\mathbf{0}$ & $0(2)$ & $0(12)$ & 0 & 20 & 0 & 0 & 0 & 0 & 0 & 0 \\
\hline Motility & 100 & 100 & $89(8)$ & $\mathrm{ND}^{c}$ & 100 & 100 & 100 & $100(30)$ & $100(3)$ & 50 & $100(1)$ \\
\hline Fluorescence & 0 & 0 & $0(12)$ & 0 & 0 & 0 & 0 & $0(27)$ & 0 & 0 & 0 \\
\hline $\begin{array}{l}\text { Growth at: } \\
5^{\circ} \mathrm{C}\end{array}$ & & & & & & & & & & & \\
\hline $10^{\circ} \mathrm{C}$ & $\begin{array}{l}100(8) \\
100\end{array}$ & $100(2)$ & $82(11)$ & $\begin{array}{l}100 \\
100\end{array}$ & $\begin{array}{r}20 \\
100\end{array}$ & $\begin{array}{r}0 \\
67\end{array}$ & $\begin{array}{r}0 \\
100\end{array}$ & $\begin{array}{c}9 \\
84(32)\end{array}$ & $\begin{array}{r}25 \\
100\end{array}$ & $\begin{array}{r}0 \\
50\end{array}$ & $\begin{array}{l}100 \\
100\end{array}$ \\
\hline $15^{\circ} \mathrm{C}$ & 100 & 100 & 100 & 100 & 100 & 100 & 100 & $100(29)$ & 100 & $100(1)$ & 100 \\
\hline $42^{\circ} \mathrm{C}$ & 100 & $50(2)$ & 69 & 100 & 100 & 100 & 100 & 100 & $100(3)$ & 100 & $50(2)$ \\
\hline $44^{\circ} \mathrm{C}$ & 11 & 0 & $25(12)$ & ND & 100 & 100 & 100 & $77(31)$ & 100 & 50 & 0 \\
\hline pH 4.5 & 63(8) & o & $33(8)$ & ND & 60 & $O(1)$ & 0 & $7(14)$ & $33(3)$ & $\mathbf{0 ( 1 )}$ & $100(2)$ \\
\hline pH 9.0 & 100 & 100 & 92 & 100 & 100 & 100 & 100 & 100 & 100 & 100 & 100 \\
\hline pH 9.5 & ND & 100 & 100 & 50 & ND & 100 & ND & 97 & 100 & 100 & 100 \\
\hline \multicolumn{12}{|l|}{ Growth on: } \\
\hline $0 \% \mathrm{NaCl}$ & $0(8)$ & $0(2)$ & 0 & 0 & 100 & 100 & 100 & $100(32)$ & $100(3)$ & 100 & $100(2)$ \\
\hline & $\mathbf{0}$ & $100(2)$ & 92 & 100 & $\mathbf{0}$ & 33 & $\mathbf{0}$ & 9 & 25 & 50 & 33 \\
\hline $10 \% \mathrm{NaCl}$ & $\mathbf{0}$ & $\mathbf{0}$ & 8 & 50 & $\mathbf{0}$ & $\mathbf{0}$ & $\mathbf{0}$ & $\mathbf{0}$ & 0 & $\mathbf{0}$ & $\mathbf{0}$ \\
\hline $\begin{array}{l}\text { Sodium lauryl } \\
\text { sulfate }\end{array}$ & 89 & 100 & 100 & 100 & 100 & 100 & 100 & 97 & 100 & 100 & 100 \\
\hline Crystal violet & $50(6)$ & 100 & 100 & 100 & 100 & 100 & $100(1)$ & 97 & 100 & 100 & 100 \\
\hline Brilliant green & 89 & 100 & 100 & 100 & 80 & 100 & 50 & 93(30) & 100 & $100(1)$ & 100 \\
\hline TCBS agar & 100 & 100 & 100 & 100 & 100 & 100 & 100 & 100 & 100 & 100 & 100 \\
\hline SS agar & 56 & 100 & 46 & 100 & 100 & 100 & 50 & 100 & 100 & 100 & 100 \\
\hline \multicolumn{12}{|l|}{ Resistant to: } \\
\hline nicin & 11 & 0 & $0(12)$ & $0(1)$ & 20 & $0(2)$ & 0 & 0 & 0 & 0 & 0 \\
\hline Peni & 89 & $\mathbf{0}$ & $67(12)$ & $100(1)$ & 100 & $100(2)$ & 0 & 9 & $\mathbf{0}$ & 100 & $\mathbf{0}$ \\
\hline $\begin{array}{l}\text { Chlorampheni- } \\
\text { col }\end{array}$ & $\mathbf{0}$ & $\mathbf{0}$ & $0(12)$ & $0(1)$ & $\mathbf{0}$ & $0(2)$ & $\mathbf{0}$ & 3 & 0 & $\mathbf{0}$ & $\mathbf{0}$ \\
\hline Erythromycin & 11 & 0 & $10(10)$ & $0(1)$ & $\mathbf{0}$ & $0(2)$ & 0 & 0 & 0 & $0(1)$ & $\mathbf{0}$ \\
\hline Kan & 0 & $\mathbf{0}$ & & o(1) & 0 & $0(2)$ & 0 & 3 & 0 & $0(1)$ & 0 \\
\hline $\begin{array}{l}\text { Chlortetracy- } \\
\text { cline }\end{array}$ & ND & $\mathbf{0}$ & $0(12)$ & $0(1)$ & ND & $100(2)$ & ND & $3(31)$ & 0 & 0 & 0 \\
\hline Novobiocin & 0 & $\mathbf{0}$ & $8(12)$ & $0(1)$ & $\mathbf{0}$ & $0(2)$ & 0 & 0 & 0 & 0 & 0 \\
\hline Polymyxin B & 67 & 100 & $83(12)$ & $100(1)$ & 100 & $100(2)$ & 100 & 73 & 100 & 100 & 33 \\
\hline $\begin{array}{l}\text { Oxytetracy- } \\
\text { cline }\end{array}$ & $0(8)$ & 0 & 0 & $0(1)$ & 20 & $100(2)$ & 0 & 0 & 0 & $0(1)$ & 0 \\
\hline Tetracycline & 11 & $\mathbf{0}$ & 8 & o(1) & $\mathbf{0}$ & $100(2)$ & 100 & $\mathbf{0}$ & 0 & $0(1)$ & $\mathbf{0}$ \\
\hline Ampicillin & 56 & 0 & $42(12)$ & $100(1)$ & 100 & $100(2)$ & 0 & 3 & 25 & $100(1)$ & $\mathbf{0}$ \\
\hline Streptomycin & ND & 33 & $0(12)$ & $0(1)$ & ND & $0(2)$ & ND & 6 & 0 & 0 & 0 \\
\hline $\begin{array}{l}\text { Susceptible to vi- } \\
\text { briostatic agent } \\
0 / 129\end{array}$ & $100(8)$ & 100 & 100 & 100 & 80 & 100 & 100 & $100(32)$ & 25 & 100 & 67 \\
\hline
\end{tabular}


TABLE 3-Continued

\begin{tabular}{|c|c|c|c|c|c|c|c|c|c|c|c|}
\hline \multirow[b]{2}{*}{ Determination } & \multicolumn{11}{|c|}{$\%$ of strains positive in phenon: } \\
\hline & $\begin{array}{c}1 \\
(n=9)\end{array}$ & $\begin{array}{c}2 \\
(n=3)\end{array}$ & $\begin{array}{c}3 \\
(n=13)\end{array}$ & $\begin{array}{c}4 \\
(n=2)\end{array}$ & $\begin{array}{c}5 \\
(n=5)\end{array}$ & $\begin{array}{c}6 \\
(n=3)\end{array}$ & $\begin{array}{c}7 \\
(n=2)\end{array}$ & $\begin{array}{c}8 \\
(n=33)\end{array}$ & $\begin{array}{c}9 \\
(n=4)\end{array}$ & $\begin{array}{c}10 \\
(n=2)\end{array}$ & $\begin{array}{c}11 \\
(n=3)\end{array}$ \\
\hline iochemical tests & & & & & & & & & & & \\
\hline Cate & 78 & 100 & 100 & 100 & 100 & 100 & 50 & 100 & 100 & 100 & $100(2)$ \\
\hline Oxidase & 100 & 67 & 100 & 100 & 100 & 100 & 100 & 100 & 100 & 100 & $100(2)$ \\
\hline $\begin{array}{l}\text { Arginine dihy- } \\
\text { drolase }\end{array}$ & 22 & 100 & 92 & 50 & 0 & $\mathbf{0}$ & 0 & 0 & 100 & 0 & 33 \\
\hline $\begin{array}{l}\text { Histidine de- } \\
\text { carboxylase }\end{array}$ & $\mathbf{0}$ & $\mathbf{0}$ & $\mathbf{0}$ & $\mathbf{0}$ & $\mathbf{0}$ & 0 & 50 & $\mathbf{0}$ & 0 & $\mathbf{0}$ & $\mathbf{0}$ \\
\hline $\begin{array}{l}\text { Lysine decar- } \\
\text { boxylase }\end{array}$ & 0 & 33 & 23 & 0 & 100 & 100 & 100 & 100 & 100 & 100 & 67 \\
\hline $\begin{array}{l}\text { Ornithine de- } \\
\text { carboxylase }\end{array}$ & $\mathbf{0}$ & $\mathbf{0}$ & 15 & $\mathbf{0}$ & 100 & $100(2)$ & 100 & 100 & 50 & 100 & 33 \\
\hline $\mathrm{H}_{2} \mathrm{~S}$ & $0(8)$ & $\mathbf{0}$ & 0 & 0 & 0 & 0 & 0 & 0 & 0 & 0 & 0 \\
\hline Methyl red & $17(6)$ & 0 & 15 & 0 & 0 & $\mathbf{0}$ & 0 & 24 & 100 & 0 & 33 \\
\hline $\begin{array}{l}\text { Voges-Pros- } \\
\text { kauer }\end{array}$ & $43(7)$ & 33 & $58(12)$ & $\mathbf{0}$ & 100 & 33 & 100 & 73 & 50 & 100 & 0 \\
\hline $\begin{array}{c}\text { Phenylalanine } \\
\text { deaminase }\end{array}$ & $0(8)$ & $\mathbf{0}$ & 0 & $\mathbf{0}$ & $\mathbf{0}$ & $\mathbf{0}$ & $\mathbf{0}$ & $\mathbf{0}$ & $0(2)$ & $\mathbf{0}$ & $\mathbf{0}$ \\
\hline $\begin{array}{l}\text { Nitrate reduc- } \\
\text { tion }\end{array}$ & $100(8)$ & 67 & 100 & 0 & 100 & 100 & 100 & 100 & 100 & 100 & 100 \\
\hline $\begin{array}{l}\text { Nitrite reduc- } \\
\text { tion }\end{array}$ & ND & 67 & 100 & $\mathbf{0}$ & ND & 100 & ND & 100 & 100 & 100 & 100 \\
\hline Phosphatase & $100(8)$ & 100 & 100 & 100 & 100 & 100 & 100 & $100(32)$ & 100 & 100 & 100 \\
\hline Ind & $50(8)$ & $\mathbf{0}$ & 39 & 0 & 100 & 100 & 100 & 91 & 100 & 50 & 67 \\
\hline Lev & 0 & 0 & $33(12)$ & 0 & 0 & 0 & 0 & $0(18)$ & 0 & 0 & 0 \\
\hline Mel & $25(8)$ & 0 & 0 & $\mathbf{0}$ & 0 & 0 & 0 & $0(32)$ & $\mathbf{0}$ & 0 & 0 \\
\hline $\begin{array}{l}\text { Iodoacetate, } \\
\text { growth }\end{array}$ & 0 & 33 & 92 & 100 & $\mathbf{0}$ & $100(1)$ & $\mathbf{0}$ & $70(30)$ & $100(3)$ & $\mathbf{0}$ & $100(2)$ \\
\hline $\begin{array}{l}\text { Iodoacetate, } \\
\text { acid }\end{array}$ & 0 & 0 & $\mathbf{0}$ & 0 & 0 & $0(1)$ & 0 & $0(30)$ & $0(3)$ & 0 & $0(2)$ \\
\hline $\begin{array}{l}\text { Alpha-hemoly- } \\
\text { sis }\end{array}$ & $\mathbf{0}$ & 0 & $0(12)$ & ND & 0 & 0 & $\mathbf{0}$ & $0(32)$ & 25 & 0 & 0 \\
\hline Beta-hemolysis & 11 & $\mathbf{0}$ & $75(12)$ & ND & 40 & 100 & 100 & $100(32)$ & 50 & 100 & 100 \\
\hline $\begin{array}{l}\text { Simmons ci- } \\
\text { trate }\end{array}$ & $67(6)$ & 67 & $100(9)$ & ND & 80 & $100(1)$ & 100 & $100(13)$ & $100(2)$ & 100 & $100(2)$ \\
\hline $\begin{array}{l}\text { Ammonia pro- } \\
\text { duction }\end{array}$ & 100 & ND & ND & ND & 100 & ND & 100 & ND & ND & ND & ND \\
\hline $\begin{array}{c}\text { Fermentative } \\
\text { metabolism }\end{array}$ & 100 & 100 & 100 & 100 & 100 & 100 & 100 & 100 & 100 & 100 & $100(1)$ \\
\hline Acid & & & & & & & & & & & \\
\hline & 22 & 0 & 85 & 0 & 0 & 0 & 0 & 0 & 50 & $\mathbf{0}$ & 33 \\
\hline & 0 & $\mathbf{0}$ & 8 & $\mathbf{0}$ & 0 & 0 & 0 & $\mathbf{0}$ & 0 & $\mathbf{0}$ & 0 \\
\hline $\mathrm{Ce}$ & $71(7)$ & 0 & 77 & 100 & 75 & $33(4)$ & 100 & 18 & 100 & 50 & 0 \\
\hline & 89 & 67 & 100 & 100 & 100 & 100 & 100 & 55 & 100 & 100 & 100 \\
\hline & 0 & 0 & 0 & 0 & 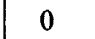 & 0 & a & 0 & 0 & 0 & 0 \\
\hline Et & 0 & 0 & 0 & 0 & 40 & 0 & 0 & 3 & 0 & 0 & 0 \\
\hline & $100(7)$ & 100 & 100 & 100 & 80 & 100 & 50 & 100 & 100 & 100 & 100 \\
\hline & $100(8$ & 67 & 92 & & 100 & 3 & 5 & $84(32)$ & 100 & 100 & 100 \\
\hline & 89 & 100 & 62 & 100 & 100 & 100 & 100 & 42 & 100 & 50 & 100 \\
\hline & 0 & 0 & 0 & 0 & 0 & 0 & 0 & 0 & 0 & 0 & 33 \\
\hline & $0(8)$ & 0 & 15 & 0 & 0 & 0 & 100 & 0 & 0 & 0 & 0 \\
\hline Mann & $100(8)$ & 100 & 100 & 100 & 100 & 100 & 100 & 100 & 100 & 100 & 100 \\
\hline & 89 & 100 & 100 & 100 & 0 & 33 & 0 & 67 & 100 & 100 & 100 \\
\hline & 0 & 0 & 0 & $\mathbf{0}$ & 0 & 0 & 0 & $\mathbf{0}$ & 0 & 0 & 0 \\
\hline & 0 & 0 & 0 & 0 & 0 & 0 & 0 & 0 & 0 & 0 & 0 \\
\hline $\mathrm{Rh}$ & $0(8$ & 0 & 8 & 0 & $\mathbf{0}$ & 0 & 0 & 0 & 0 & 0 & 0 \\
\hline & 0 & 0 & 0 & 50 & 0 & 0 & 0 & 0 & 0 & 0 . & 33 \\
\hline Sor & 44 & 100 & 77 & 0 & 0 & 0 & 0 & 6 & 25 & $\mathbf{0}$ & 0 \\
\hline Sorbo & 0 & 0 & 0 & 0 & 0 & 0 & 0 & 0 & 0 & 0 & 0 \\
\hline Sucr & $100(6)$ & $100(2)$ & 100 & 50 & 100 & 100 & 100 & 73 & 50 & 100 & 100 \\
\hline Trehalose & 100 & 100 & 100 & 100 & 40 & 100 & $0(1)$ & 91 & 100 & 100 & 100 \\
\hline Xylose & $\mathbf{0}$ & 0 & 8 & 0 & 0 & 0 & 0 & 0 & 0 & 0 & 0 \\
\hline
\end{tabular}


TABLE 3-Continued

\begin{tabular}{|c|c|c|c|c|c|c|c|c|c|c|c|}
\hline \multirow[b]{2}{*}{ Determination } & \multicolumn{11}{|c|}{ \% of strains positive in phenon: } \\
\hline & $\begin{array}{c}1 \\
(n=9)\end{array}$ & $\begin{array}{c}2 \\
(n=3)\end{array}$ & $\begin{array}{c}3 \\
(n=13)\end{array}$ & $\begin{array}{c}4 \\
(n=2)\end{array}$ & $\begin{array}{c}5 \\
(n=5)\end{array}$ & $\begin{array}{c}6 \\
(n=3)\end{array}$ & $\begin{array}{c}7 \\
(n=2)\end{array}$ & $\begin{array}{c}8 \\
(n=33)\end{array}$ & $\begin{array}{c}9 \\
(n=4)\end{array}$ & $\begin{array}{c}10 \\
(n=2)\end{array}$ & $\begin{array}{c}11 \\
(n=3)\end{array}$ \\
\hline Gas from: & & & & & & & & & & & \\
\hline Glucose & 0 & 33 & 0 & 0 & 0 & 0 & 0 & $3(32)$ & 100 & 0 & 33 \\
\hline Lactose & $\mathbf{0}$ & 0 & 0 & $\mathbf{0}$ & 0 & $\mathbf{0}$ & 0 & $0(32)$ & 0 & 0 & 0 \\
\hline Degradation of: & & & & & & & & & & & \\
\hline Urea & 0 & 0 & 8 & 0 & 0 & 0 & 0 & 0 & 0 & 0 & 0 \\
\hline Starch & $86(6)$ & 100 & 100 & $100(1)$ & $100(4)$ & 100 & 100 & $72(29)$ & 75 & 100 & 100 \\
\hline Chitin & $100(1)$ & $100(2)$ & $60(10)$ & $0(1)$ & 100 & 100 & 100 & $94(32)$ & 100 & $100(1)$ & 100 \\
\hline Gelatin & $100(1)$ & ND & $100(5)$ & ND & 100 & $100(1)$ & 100 & 100 & 100 & 100 & $100(2)$ \\
\hline Casein & $88(7)$ & 100 & 100 & $0(1)$ & 100 & 100 & 100 & 100 & 100 & 100 & 100 \\
\hline DNA & $50(4)$ & 100 & 100 & 100 & 100 & 100 & 0 & 91(32) & 100 & 100 & 100 \\
\hline Tween 20 & 100 & 100 & 100 & 100 & 100 & 100 & 100 & 100 & 100 & 100 & 100 \\
\hline Tween 40 & 100 & 100 & 100 & 100 & 100 & 100 & 100 & $100(31)$ & 100 & 100 & 100 \\
\hline Tween 60 & 100 & 100 & 100 & 100 & 100 & 100 & 100 & 100 & 100 & 100 & 100 \\
\hline Tween 80 & $100(8)$ & 100 & 100 & 100 & 100 & 100 & 100 & 100 & 100 & 100 & 100 \\
\hline Lecithin & $50(8)$ & 100 & $100(10)$ & 100 & 80 & 67 & 50 & 94 & 75 & 100 & 100 \\
\hline Tyrosine & 33 & 0 & 8 & 0 & 0 & 0 & 0 & $3(32)$ & 0 & 0 & $0(2)$ \\
\hline Allantoin & 0 & ND & ND & ND & 0 & ND & 0 & ND & ND & ND & ND \\
\hline Chondroitin & ND & $0(1)$ & $60(5)$ & $100(1)$ & ND & 67 & ND & 21(19) & $50(2)$ & 0 & $100(1)$ \\
\hline $\begin{array}{l}\text { Esculin (by } \\
\text { color) }\end{array}$ & $0(6)$ & 0 & 8 & 50 & $0(2)$ & 0 & $\mathbf{0}$ & 0 & 0 & 0 & 67 \\
\hline Esculin (by & $0(6)$ & 0 & 0 & 0 & $0(1)$ & 0 & 0 & 0 & 0 & 0 & $50(2)$ \\
\hline UV light) & & & & & & & & & & & \\
\hline $\begin{array}{r}\text { Utilization as } \\
\text { sole carbon }\end{array}$ & & & & & & & & & & & \\
\hline Acetate & 89 & $100(1)$ & $18(11)$ & 0 & 100 & 0 & 50 & $50(30)$ & 50 & 0 & 0 \\
\hline Beta-alanine & 0 . & ND & $0(8)$ & 0 & 0 & $0(1)$ & 0 & $0(2)$ & ND & ND & $0(1)$ \\
\hline DL-Alanine & 100 & 100 & 100 & 100 & 100 & 67 & 100 & 88 & 100 & 100 & 100 \\
\hline Alginate & ND & 0 & 0 & 50 & ND & 0 & ND & 0 & 0 & 0 & 0 \\
\hline Arginine & 89 & 0 & 92 & 100 & 100 & 33 & 100 & $97(30)$ & 100 & $100(1)$ & 100 \\
\hline Citrate & 100 & 100 & 100 & 100 & 100 & 100 & 100 & 94 & 100 & 100 & 100 \\
\hline Formate & 0 & 0 & 0 & 0 & 0 & 0 & 0 & 0 & 0 & 0 & 0 \\
\hline Glycine & 0 & 0 & $17(12)$ & 100 & 100 & 33 & 100 & 18 & 75 & 0 & 33 \\
\hline Inulin & 100 & 100 & 92 & 100 & 100 & 67 & 50 & 97 & 100 & 100 & 100 \\
\hline Lysine & 0 & 0 & 0 & 0 & 0 & 0 & 0 & 3 & 0 & 0 & 0 \\
\hline Malonate & 0 & 0 & 0 & 0 & 0 & 0 & 0 & 0 & 0 & 0 & 0 \\
\hline Phenylalanine & 0 & 0 & 8 & 0 & 0 & 0 & 0 & 12 & $0(3)$ & 0 & 0 \\
\hline Proline & 67 & 100 & 100 & 100 & 100 & 100 & 100 & 91 & 100 & 100 & 100 \\
\hline Propanol & 0 & 0 & 8 & $\mathbf{0}$ & 0 & 0 & $\mathbf{0}$ & 6 & 75 & $\mathbf{0}$ & 0 \\
\hline Pyruvate & 100 & $100(2)$ & 100 & 100 & 100 & 67 & 100 & $97(32)$ & 100 & 0 & 100 \\
\hline Serine & 100 & 100 & 69 & 100 & 100 & 100 & 100 & $78(32)$ & 100 & 50 & 100 \\
\hline Succinate & 100 & 33 & 54 & 100 & 80 & 100 & 100 & $23(31)$ & 75 & 0 & 33 \\
\hline Tartrate & 0 & 0 & 23 & 50 & 0 & 0 & 0 & 3 & 0 & 0 & 0 \\
\hline Tryptophan & 0 & 0 & 15 & $\mathbf{0}$ & $0(3)$ & 33 & $\mathbf{0}$ & $\mathbf{0}$ & $\mathbf{0}$ & $\mathbf{0}$ & 0 \\
\hline Uracil & 0 & 0 & 0 & 0 & 0 & 0 & 0 & 0 & 0 & 0 & 0 \\
\hline
\end{tabular}

${ }^{a}$ All phena were positive for the following tests: growth at $37^{\circ} \mathrm{C}$ and $\mathrm{pH} 8$, growth on $3 \% \mathrm{NaCl}$, neutral red, and pyronin $G$, and rod-shaped cells. All phena were negative for production of acid from adonitol, dulcitol, amygdalin, and melizitose, resistance to nalidixic acid, cellulose hydrolysis, and formation of chains.

${ }^{b}$ The numbers in parentheses indicate the numbers of strains tested for different traits.

c ND, Not done.

ture. Cluster 1 strains did not grow in media containing $7 \% \mathrm{NaCl}$, whereas strains in clusters 2 through 4 did grow at this salinity. We also observed differences in the production of acid from arabinose, cellobiose, and sorbitol and in the utilization of acetate and arginine. The clus- ter 4 strains differed from the strains of the other three clusters in that they were negative for chitin hydrolysis, casein hydrolysis, and nitrate reduction.

The strains in clusters 5 through 8 were all identified as members of $V$. cholerae. Cluster 5 
consisted of five strains, all of which were isolated from Copenhagen sewage. Cluster 6 contained three strains, strain DAM12/75 from Copenhagen sewage and two strains of $V$. cholerae received from R. Sakazaki (strain 10317-74 [serovar 8] and strain 11416-62 [serovar 13]). Two additional reference strains of $V$. cholerae were recovered in cluster 7 ; these were strain 7007-64 (serovar 6) and strain 4202 (serovar 5). Cluster 8 was the largest cluster recovered in the analysis. This cluster contained 33 strains, including 19 strains of $V$. cholerae isolated from the Chesapeake Bay (strains V2, V3, V35, V33, V31, V37, V4, V5, V10, V36, V38, V11, V15, V25, V26, V20, V24, V19, and V29), 3 strains of $V$. cholerae received from R. Sakazaki (strains 211-72, 218-68, and 8645-64 [serovars 12, 10, and 14 , respectively]), 9 strains of $V$. cholerae non01 received from $H$. Smith and isolated in Thailand, in Czechoslovakia, from the Chesapeake Bay, and in the Philippines, 1 strain of $V$. cholerae O1 (strain Inaba, isolated in Bangladesh), and 2 reference strains of $V$. cholerae 01 obtained from the American Type Culture Collection (ATCC 14033 and ATCC 14035, representing the El Tor and classical biovars, respectively).

The strains of clusters 5 through 8 exhibited characteristics ascribed to $V$. cholerae; i.e., they were gram-negative, facultatively anaerobic, rod-shaped organisms that were oxidase positive, susceptible to vibriostatic agent $0 / 129$, and motile and produced acid but not gas from glucose and mannitol, but not from arabinose, inositol, or xylose. These strains were positive for nitrate reduction, lysine decarboxylase, ornithine decarboxylase, growth in 0 and $3 \% \mathrm{NaCl}$, hydrolysis of Tween, gelatin, starch, and casein, and indole production, but negative for arginine dihydrolase and growth in $7 \% \mathrm{NaCl}$. The guanine-plus-cytosine $(\mathrm{G}+\mathrm{C})$ contents of the DNAs of selected strains were 47 to $48 \mathrm{~mol} \%$ (16), values typical of $V$. cholerae (82).

Certain differences were observed among clusters 5 through 8. For example, antibiotic resistance patterns revealed that strains of clusters 5 and 6 were ampicillin resistant, whereas strains of clusters 7 and 8 were ampicillin susceptible; strains of clusters 6 and 7 were tetracycline resistant, and strains of cluster 7 were also resistant to oxytetracycline.

We also observed differences in the utilization of sole carbon sources; strains of clusters 5 and 7 utilized glycine, whereas strains of clusters 6 and 8 were not able to utilize this compound. Strains of cluster 5 were uniformly positive for acetate utilization, whereas strains of cluster 6 were negative and strains of the remaining two clusters were variable for utilization of this carbon source. Other notable exceptions in characteristics include the lack of deoxyribonuclease activity in strains of cluster 7 and the production of colorless colonies by strains of cluster 5 . Acid production from certain carbohydrates also revealed differences; strains of cluster 7 were lactose positive, whereas the other strains were negative for this characteristic.

Strains of cluster 9 were identified as being members of Aeromonas hydrophila; these were strains V16 and V22 from the Chesapeake Bay, strain K204V from seawater in Denmark, and strain 112-68, which was received from $R$. Sakazaki as $V$. cholerae serovar 9 . In general, these strains adhered to the description of $A$. hydrophila provided by various investigators $(26,71$, 81). The cluster 9 strains were gram-negative, facultatively anaerobic, rod-shaped organisms which were oxidase positive and motile. They were resistant to vibriostatic agent $0 / 129$, produced acid and gas from a variety of sugars, and gave a positive reaction for arginine dihydrolase. However, strains K204V and 112-68 were ornithine positive, a characteristic which has not been reported previously for $A$. hydrophila. These two strains were unusual in that they were susceptible to vibriostatic agent $0 / 129$, which, together with the ornithine decarboxylase reaction, suggests that they are vibrios. However, these strains produced gas from glucose and acid from arabinose, characteristics which are usually negative for $V$. cholerae but positive for aeromonads.

Cluster 10 contained two strains, strain 128275 from Denmark and strain 5049, which was received from $\mathrm{H}$. Smith and was originally isolated from freshwater in Thailand. The strains in this cluster were very similar to strains in the main cluster of $V$. cholerae and possessed the major characteristics of this species (see above).

Three strains were recovered in cluster 11; two of these were from Denmark, and one, strain 10037-74, was received as Vibrio proteus from R. Sakazaki. These strains appear to be intermediate strains, belonging to neither Vibrio nor Aeromonas, as Table 4 shows. On the basis of the data gathered in this study, no definitive species assignment of the strains of phenon 11 can be made at the present time.

A total of 14 vibrio strains isolated from diseased shrimp were recovered in cluster 12, along with the reference strain of $V$. parahaemolyticus (strain ATCC 17802). Also recovered in cluster 12 was a reference culture of Beneckea harveyi. This phenon was identified as $V$. parahaemolyticus on the basis of biochemical characteristics (Table 4) and the inclusion of the type strain of this species in the cluster. The base composition of selected strains was $45 \mathrm{~mol} \%$ $\mathrm{G}+\mathrm{C}$. The biochemical characteristics of these strains agree well with the accepted definition of 
TABLE 4. Characteristics of Vibrio phena 12 through 22

\begin{tabular}{|c|c|c|c|c|c|c|c|c|c|c|c|}
\hline \multirow[b]{2}{*}{ Determination } & \multicolumn{11}{|c|}{$\%$ of strains positive in phenon: } \\
\hline & $\begin{array}{c}12 \\
(n=16)\end{array}$ & $\begin{array}{c}13 \\
(n=2)\end{array}$ & $\begin{array}{c}14 \\
(n=5)\end{array}$ & $\begin{array}{c}15 \\
(n=2)\end{array}$ & $\begin{array}{c}16 \\
(n=2)\end{array}$ & $\begin{array}{c}17 \\
(n=6)\end{array}$ & $\begin{array}{c}18 \\
(n=16)\end{array}$ & $\begin{array}{c}19 \\
(n=3)\end{array}$ & $\begin{array}{c}20 \\
(n=7)\end{array}$ & $\begin{array}{c}21 \\
(n=3)\end{array}$ & $\begin{array}{c}22 \\
(n=2)\end{array}$ \\
\hline \multicolumn{12}{|l|}{ Colonial morpholo- } \\
\hline $\begin{array}{l}\text { gy } \\
\text { White or cream }\end{array}$ & 25 & 50 & $0(4)^{a}$ & 0 & 100 & 0 & $53(15)$ & 0 & 0 & 0 & 0 \\
\hline Yellow & 0 & 0 & $0(4)$ & 0 & 0 & 67 & $7(15)$ & 0 & 0 & 0 & 100 \\
\hline Colorless & 75 & 50 & $100(4)$ & 100 & 0 & 33 & $40(15)$ & 100 & 100 & 100 & 0 \\
\hline Diffusible pigment & 0 & 0 & $0(4)$ & 0 & 0 & 0 & 0 & 0 & 0 & 0 & $\mathbf{0}$ \\
\hline Mucoid & 0 & 0 & 0 & $\mathbf{0}$ & 0 & 0 & 13(15) & 0 & 0 & 0 & 0 \\
\hline Matt & 0 & 0 & 0 & 0 & 0 & 0 & 0 & 0 & $\mathbf{0}$ & 33 & 0 \\
\hline Translucent & 94 & 100 & 100 & 100 & 100 & 100 & 63 & 100 & 100 & 67 & 50 \\
\hline Round & 100 & 100 & 100 & 100 & 100 & 100 & 100 & 100 & 100 & 100 & 100 \\
\hline Irregular margin & 0 & 0 & 0 & 0 & 0 & 0 & 0 & 0 & $\mathbf{0}$ & 0 & 0 \\
\hline Rhizoid margin & 0 & 0 & 0 & 0 & 0 & 0 & 0 & 0 & 0 & 0 & 0 \\
\hline Flat & 6 & 50 & 20 & 100 & 0 & 100 & 44 & 100 & 0 & 0 & 0 \\
\hline Spreading & 13 & 0 & 0 & 0 & 0 & 0 & 0 & 0 & 0 & 0 & 0 \\
\hline \multicolumn{12}{|l|}{ Micromorphology } \\
\hline Gram variable & $0(15)$ & 0 & $0(4)$ & 0 & $0(1)$ & 0 & 0 & 0 & 14 & $\mathbf{0}$ & 0 \\
\hline $\begin{array}{l}\text { Length/width ratio } \\
\text { of } \geq 6: 1\end{array}$ & $0(15)$ & 0 & $0(4)$ & 0 & $0(1)$ & 17 & 6 & 0 & 100 & 0 & 0 \\
\hline Filaments & $0(15)$ & 0 & $0(4)$ & 0 & $0(1)$ & 33 & 0 & 0 & 43 & 0 & 50 \\
\hline Motility & 92(13) & 100 & $100(2)$ & $100(1)$ & $100(1)$ & 100 & $100(15)$ & 100 & $100(4)$ & $100(2)$ & 100 \\
\hline Fluorescence & $0(9)$ & 0 & 0 & 0 & 0 & 0 & 0 & 0 & 0 & 0 & 0 \\
\hline \multicolumn{12}{|l|}{ Growth at: } \\
\hline $5^{\circ} \mathrm{C}$ & $0(15)$ & 0 & 0 & 0 & 0 & 100 & 100 & 100 & 86 & 100 & 50 \\
\hline $10^{\circ} \mathrm{C}$ & 94 & 100 & 100 & 100 & $100(1)$ & 100 & 100 & 100 & 100 & 100 & 100 \\
\hline $15^{\circ} \mathrm{C}$ & 94 & 100 & 100 & 100 & 100 & 100 & 100 & 100 & 100 & 100 & 100 \\
\hline $42^{\circ} \mathrm{C}$ & $100(15)$ & 100 & 100 & 100 & 100 & 100 & 92(13) & $100(2)$ & 71 & 0 & 100 \\
\hline $44^{\circ} \mathrm{C}$ & 88 & 0 & 40 & 0 & 100 & 100 & 19 & 0 & 14 & 0 & 50 \\
\hline $\mathrm{pH} 4.5$ & $0(14)$ & $\mathrm{ND}^{b}$ & 0 & $100(1)$ & $100(1)$ & $75(4)$ & 6 & 0 & $0(4)$ & 0 & $100(1)$ \\
\hline pH 9.0 & 100 & 100 & 100 & 100 & $100(1)$ & 100 & 100 & 100 & 86 & 100 & 100 \\
\hline pH 9.5 & $100(14)$ & 100 & 100 & ND & 100 & ND & $100(12)$ & 100 & $100(3)$ & $100(2)$ & ND \\
\hline \multicolumn{12}{|l|}{ Growth on: } \\
\hline $0 \% \mathrm{NaCl}$ & 0 & 0 & 0 & 0 & $100(1)$ & 17 & $93(15)$ & 100 & 100 & $0(2)$ & 50 \\
\hline $7 \% \mathrm{NaCl}$ & 100 & 100 & 60 & 0 & 100 & 100 & 100 & 100 & 100 & $50(2)$ & $100(1)$ \\
\hline $10 \% \mathrm{NaCl}$ & 0 & 0 & 0 & 0 & 50 & 0 & 0 & 0 & 0 & 0 & 0 \\
\hline $\begin{array}{l}\text { Sodium lauryl } \\
\text { sulfate }\end{array}$ & 88 & 100 & 100 & 50 & 50 & 100 & 100 & 100 & $33(6)$ & $100(2)$ & 0 \\
\hline Crystal violet & 100 & 100 & 100 & $0(1)$ & 100 & 100 & $100(15)$ & 100 & $100(5)$ & 100 & 0 \\
\hline Brilliant green & 100 & 100 & $75(4)$ & 100 & 50 & 100 & 100 & 100 & 14 & 100 & 100 \\
\hline TCBS agar & 100 & 100 & 100 & $100(1)$ & 100 & 100 & 100 & 100 & 14 & 100 & 100 \\
\hline SS agar & 81 & 100 & 0 & 50 & 0 & $100(5)$ & 94 & 100 & $0(6)$ & 33 & 100 \\
\hline \multicolumn{12}{|l|}{ Resistant to: } \\
\hline Gentamicin & $0(13)$ & 0 & $0(1)$ & 0 & 0 & 0 & 0 & 0 & 0 & 0 & 50 \\
\hline Penicillin & $100(13)$ & 100 & $100(1)$ & 100 & 0 & 100 & 6 & 33 & 0 & 0 & 100 \\
\hline Chloramphenicol & $0(13)$ & 0 & $0(1)$ & 0 & 100 & 0 & 0 & 0 & 0 & 0 & 0 \\
\hline Erythromycin & $0(12)$ & 0 & $0(1)$ & 0 & 0 & 0 & 0 & 0 & 0 & 33 & 0 \\
\hline Kanamycin & $0(13)$ & $\mathbf{0}$ & $0(1)$ & $\mathbf{0}$ & $\mathbf{0}$ & 0 & 0 & 0 & $\mathbf{0}$ & 0 & 0 \\
\hline Chlortetracycline & $0(9)$ & 0 & ND & ND & 0 & ND & $0(12)$ & 0 & $0(4)$ & 0 & ND \\
\hline Novobiocin & $0(13)$ & 0 & $0(1)$ & 0 & 0 & 0 & 0 & 0 & 0 & 0 & 0 \\
\hline Polymyxin B & $15(13)$ & 50 & $0(1)$ & 50 & 100 & $20(5)$ & 75 & 33 & 43 & 100 & 100 \\
\hline Oxytetracycline & $0(13)$ & $0(1)$ & $0(1)$ & $0(1)$ & $\mathbf{0}$ & 0 & 0 & 0 & 0 & 0 & 0 \\
\hline Tetracycline & $0(13)$ & 0 & $0(1)$ & $0(1)$ & 0 & 0 & $\mathbf{0}$ & $\mathbf{0}$ & 0 & $\mathbf{0}$ & 0 \\
\hline Ampicillin & $92(13)$ & 50 & $100(1)$ & 50 & 0 & 50 & 6 & 67 & 0 & 0 & 100 \\
\hline Streptomycin & $0(12)$ & 0 & $0(1)$ & ND & 0 & ND & $8(12)$ & 0 & $0(4)$ & $\mathbf{0}$ & ND \\
\hline $\begin{array}{l}\text { Susceptible to vi- } \\
\text { briostatic agent } 0 / \\
129\end{array}$ & $15(13)$ & 0 & 100 & 100 & 50 & 100 & $100(13)$ & 100 & 100 & 100 & 50 \\
\hline \multicolumn{12}{|l|}{ Biochemical tests } \\
\hline Catalase & 100 & 100 & $100(4)$ & 100 & 100 & 100 & 100 & 100 & 100 & 100 & 100 \\
\hline Oxidase & 100 & 100 & $100(4)$ & 100 & 100 & 0 & 0 & 0 & 0 & 100 & 100 \\
\hline $\begin{array}{l}\text { Arginine dihydro- } \\
\text { lase }\end{array}$ & $0(15)$ & 0 & $0(2)$ & 100 & 100 & 0 & 31 & 0 & 0 & 100 & 0 \\
\hline $\begin{array}{l}\text { Histidine decar- } \\
\text { boxylase }\end{array}$ & $7(15)$ & $\mathbf{0}$ & 0 & 0 & 0 & 33 & $\mathbf{0}$ & 0 & 43 & $\mathbf{0}$ & 50 \\
\hline
\end{tabular}


TABLE 4-Continued

\begin{tabular}{|c|c|c|c|c|c|c|c|c|c|c|c|}
\hline \multirow[b]{2}{*}{ Determination } & \multicolumn{11}{|c|}{$\%$ of strains positive in phenon: } \\
\hline & $\begin{array}{c}12 \\
(n=16)\end{array}$ & $\left(\begin{array}{c}13 \\
(n=2)\end{array}\right.$ & $\begin{array}{c}14 \\
(n=5)\end{array}$ & $\begin{array}{c}15 \\
(n=2)\end{array}$ & $\begin{array}{c}16 \\
(n=2) \\
\end{array}$ & $\begin{array}{c}17 \\
(n=6)\end{array}$ & $\begin{array}{c}18 \\
(n=16)\end{array}$ & $\begin{array}{c}19 \\
(n=3)\end{array}$ & $\begin{array}{c}20 \\
(n=7)\end{array}$ & $\begin{array}{c}21 \\
(n=3)\end{array}$ & $\begin{array}{c}22 \\
(n=2)\end{array}$ \\
\hline $\begin{array}{l}\text { Lysine decarbox- } \\
\text { ylase }\end{array}$ & $100(15)$ & 100 & 40 & 50 & 50 & $80(5)$ & 75 & 67 & 71 & 0 & 50 \\
\hline $\begin{array}{l}\text { Ornithine decar- } \\
\text { boxylase }\end{array}$ & 93(15) & 100 & $100(1)$ & $\mathbf{0}$ & 50 & $\mathbf{0}$ & $\mathbf{0}$ & $\mathbf{0}$ & 14 & 0 & 100 \\
\hline $\mathrm{H}_{2} \mathrm{~S}$ & $\mathbf{0}$ & 50 & 0 & $0(1)$ & $\mathbf{0}$ & $\mathbf{0}$ & $\mathbf{0}$ & $\mathbf{0}$ & $\mathbf{0}$ & 0 & 0 \\
\hline Methyl red & 100 & 100 & 40 & ND & $\mathbf{0}$ & 83 & $20(15)$ & $\mathbf{0}$ & $0(6)$ & $\mathbf{0}$ & $\mathbf{0}$ \\
\hline Voges-Proskauer & $\mathbf{0}$ & $\mathbf{0}$ & 0 & 50 & 100 & 67 & 73(15) & 67 & 86 & 100 & $100(1)$ \\
\hline $\begin{array}{l}\text { Phenylalanine de- } \\
\text { aminase }\end{array}$ & 13(15) & $\mathbf{0}$ & $\mathbf{0}$ & $0(1)$ & 0 & $\mathbf{0}$ & $\mathbf{0}$ & 0 & $\mathbf{0}$ & 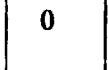 & 0 \\
\hline Nitrate reduction & $100(15)$ & 100 & 67(3) & $100(1)$ & 100 & 0 & 0 & $\mathbf{0}$ & 14 & $100(2)$ & 100 \\
\hline Nitrite reduction & $100(15)$ & 100 & $67(3)$ & ND & 100 & 0 & $0(15)$ & $\mathbf{0}$ & $0(6)$ & $100(2)$ & ND \\
\hline Phosphatase & & 100 & $100(4)$ & ND & 100 & 100 & $87(15)$ & 100 & $67(6)$ & 100 & 100 \\
\hline Indole & $83(12)$ & 100 & $100(4)$ & $\mathbf{0}$ & $\mathbf{0}$ & 17 & 19 & $\mathbf{0}$ & 57 & $\mathbf{0}$ & 100 \\
\hline Levan & $O(2)$ & ND & ND & $\mathbf{0}$ & $\mathbf{0}$ & 0 & $\mathbf{0}$ & $\mathbf{0}$ & 0 & $\mathbf{0}$ & 0 \\
\hline Melanin & $0(2)$ & $\mathbf{0}$ & $0(2)$ & 0 & 0 & 17 & $\mathbf{0}$ & $\mathbf{0}$ & $\mathbf{0}$ & 0 & 0 \\
\hline $\begin{array}{l}\text { Iodoacetate, } \\
\text { growth }\end{array}$ & 100 & 100 & 100 & 0 & 100 & 0 & 69 & 67 & $0(3)$ & 67 & $0(1)$ \\
\hline Iodoacetate, acid & 0 & 0 & $\mathbf{0}$ & $\mathbf{0}$ & 0 & 0 & $\mathbf{0}$ & $\mathbf{0}$ & o(3) & 0 & $0(1)$ \\
\hline Alpha-hemolysis & 64(14) & 50 & $0(4)$ & $\mathbf{0}$ & $0(1)$ & $\mathbf{0}$ & 6 & $\mathbf{0}$ & $\mathbf{0}$ & $\mathbf{0}$ & 0 \\
\hline Beta-hemolysis & $0(14)$ & $\mathbf{0}$ & $50(4)$ & 50 & $0(1)$ & 33 & 81 & 100 & 57 & 67 & 50 \\
\hline Simmons citrate & ND & ND & ND & $0(1)$ & $0(1)$ & 100 & 81 & 100 & $75(4)$ & $100(2)$ & $100(1)$ \\
\hline $\begin{array}{l}\text { Ammonia produc- } \\
\text { tion }\end{array}$ & ND & ND & ND & 100 & ND & 100 & $100(14)$ & ND & $100(4)$ & ND & 100 \\
\hline $\begin{array}{l}\text { Fermentative me- } \\
\text { tabolism }\end{array}$ & $100(15)$ & 100 & 40 & 100 & 50 & 100 & 100 & 100 & $100(5)$ & 100 & 100 \\
\hline \multicolumn{12}{|l|}{ Acid from: } \\
\hline Arabinose & 31 & 100 & 0 & $0(1)$ & 0 & $0(5)$ & $0(15)$ & $\mathbf{0}$ & $\mathbf{0}$ & 33 & $\mathbf{0}$ \\
\hline Butanc & 0 & 0 & 0 & 0 & 0 & 0 & 0 & 0 & 0 & 0 & 0 \\
\hline Cellobiose & 100 & 50 & 60 & $0(1)$ & 100 & 33 & 38 & 0 & 0 & 100 & 100 \\
\hline Dextrin & 50 & 100 & 0 & $0(1)$ & 50 & 100 & 100 & 100 & 100 & 100 & 50 \\
\hline Ery & 0 & 0 & 0 & 0 & 0 & 0 & 0 & 0 & 0 & 0 & 0 \\
\hline Ethanol & 0 & 0 & 0 & 0 & 0 & 0 & 0 & 0 & 0 & 0 & 0 \\
\hline Fructose & 100 & 100 & 100 & $100(1)$ & 100 & 100 & 100 & 100 & $100(6)$ & 100 & 100 \\
\hline Galactose & 94 & 100 & 100 & $100(1)$ & 50 & 67 & \begin{tabular}{|l|}
94 \\
\end{tabular} & 0 & 100 & 100 & 100 \\
\hline Glycerol & 75 & 50 & 60 & $100(1)$ & 0 & 100 & 100 & 100 & 86 & 100 & 100 \\
\hline Inositol & 0 & 0 & 0 & 0 & 0 & 33 & $\begin{array}{l}69 \\
\end{array}$ & 0 & 100 & 0 & 0 \\
\hline Lactose & 0 & 0 & 0 & 0 & 0 & 67 & 88 & 0 & 43 & 0 & 0 \\
\hline Mannitol & 94 & 100 & 80 & $0(1)$ & $100(1)$ & 100 & 100 & 100 & $100(6)$ & 100 & 100 \\
\hline Mannose & 93(14) & 100 & 40 & 100 & 100 & $100(5)$ & 88 & 100 & 100 & 100 & 50 \\
\hline Melibiose & 0 & 0 & 0 & $0(1)$ & 0 & 67 & 94 & 0 & 0 & 0 & 0 \\
\hline Raffin & 0 & 0 & 0 & 0 & 0 & 17 & 0 & 0 & 0 & o & 0 \\
\hline Rhamnose & 0 & 0 & 0 & $\mathbf{0}$ & 0 & 0 & 0 & 0 & 0 & 0 & 0 \\
\hline Salic & 0 & 0 & 20 & 0 & 0 & 0 & 13 & 0 & 0 & 0 & 0 \\
\hline Sorbitol & $0(14)$ & 0 & $33(3)$ & 0 & o & 33 & 38 & o & 0 & o & 50 \\
\hline Sorbose & 0 & 0 & 0 & $\mathbf{0}$ & $\mathbf{0}$ & 0 & 0 & 0 & 0 & 0 & 0 \\
\hline Sucrose & 94 & 0 & 100 & $0(1)$ & 100 & 100 & $100(14)$ & 100 & 100 & 100 & $100(1)$ \\
\hline Trehalose & 100 & 100 & $100(4)$ & 100 & 100 & 100 & 100 & 100 & 100 & 100 & 50 \\
\hline Xylose & 0 & 0 & ND & 0 & 0 & 0 & 6 & 0 & 0 & 0 & 0 \\
\hline \multicolumn{12}{|l|}{ Gas from: } \\
\hline Glucose & 13(8) & 0 & $0(2)$ & 0 & $\mathbf{0}$ & 0 & $7(15)$ & 0 & 0 & $O(2)$ & 0 \\
\hline Lactc & 0 & 0 & 0 & 0 & $\mathbf{0}$ & 0 & $7(15)$ & 0 & 0 & $0(2)$ & 0 \\
\hline \multicolumn{12}{|l|}{ Degradation of: } \\
\hline Urea & 81 & $\mathbf{0}$ & 0 & 50 & $\mathbf{0}$ & 0 & $\mathbf{0}$ & 0 & $\mathbf{0}$ & $\mathbf{0}$ & 0 \\
\hline Starch & 100 & 100 & $100(4)$ & $100(1)$ & 100 & $100(3)$ & 100 & 100 & $100(5)$ & 100 & 100 \\
\hline Chitin & $73(15)$ & 100 & $100(4)$ & ND & $0(1)$ & $100(4)$ & $100(15)$ & 100 & $100(6)$ & 100 & $100(1)$ \\
\hline Gelatin & $82(11)$ & 100 & 100 & ND & $0(1)$ & $100(3)$ & 91(11) & 100 & $83(6)$ & $100(1)$ & $100(1)$ \\
\hline Casein & $40(15)$ & 100 & $75(45)$ & 100 & 50 & 100 & 69 & ND & 100 & 100 & 100 \\
\hline DNA & $100(15)$ & 100 & $100(4)$ & $100(1)$ & 100 & $80(5)$ & $100(14)$ & $100(2)$ & $100(4)$ & 100 & $100(1)$ \\
\hline Tween 20 & 100 & 100 & 100 & 100 & 100 & 100 & 100 & $100(2)$ & $100(6)$ & 100 & 0 \\
\hline Tween 40 & 100 & 100 & 100 & 100 & $0(1)$ & 100 & 100 & 100 & 100 & 100 & 0 \\
\hline Tween 60 & 100 & 100 & & 100 & 100 & 100 & 100 & 0 & 10 & 100 & 50 \\
\hline Tween 80 & 100 & 100 & 100 & 100 & 100 & $100(5)$ & 93(15) & $100(2)$ & 100 & 100 & 0 \\
\hline Lecithin & $80(15)$ & 50 & $100(4)$ & $0(1)$ & 100 & $100(5)$ & $100(13)$ & 100 & $67(6)$ & $50(2)$ & 100 \\
\hline
\end{tabular}


TABLE 4-Continued

\begin{tabular}{|c|c|c|c|c|c|c|c|c|c|c|c|}
\hline \multirow[b]{2}{*}{ Determination } & \multicolumn{11}{|c|}{$\%$ of strains positive in phenon: } \\
\hline & $\begin{array}{c}12 \\
(n=16)\end{array}$ & $\begin{array}{c}13 \\
(n=2) \\
\end{array}$ & $\begin{array}{c}14 \\
(n=5) \\
\end{array}$ & $\begin{array}{c}15 \\
(n=2) \\
\end{array}$ & $\begin{array}{c}16 \\
(n=2)\end{array}$ & $\begin{array}{c}17 \\
(n=6)\end{array}$ & $\begin{array}{c}18 \\
(n=16)\end{array}$ & $\begin{array}{c}19 \\
(n=3)\end{array}$ & $\begin{array}{c}20 \\
(n=7)\end{array}$ & $\begin{array}{c}21 \\
(n=3) \\
\end{array}$ & $\begin{array}{c}22 \\
(n=2)\end{array}$ \\
\hline Tyrosine & $0(14)$ & $\mathbf{0}$ & $0(2)$ & $\mathbf{0}$ & $\mathbf{0}$ & 17 & 0 & $\mathbf{0}$ & $\mathbf{0}$ & 33 & $\mathbf{0}$ \\
\hline Allantoin & ND & ND & ND & 0 & ND & 0 & $0(14)$ & ND & $0(4)$ & ND & 0 \\
\hline Chondroitin & 69 & 0 & $25(4)$ & ND & 50 & ND & $0(1)$ & ND & $0(3)$ & $50(2)$ & ND \\
\hline $\begin{array}{l}\text { Esculin (by col- } \\
\text { or) }\end{array}$ & $7(15)$ & 0 & 100 & $0(1)$ & $100(1)$ & $75(4)$ & $87(15)$ & 67 & $100(4)$ & 0 & 0 \\
\hline $\begin{array}{l}\text { Esculin (by UV } \\
\text { light) }\end{array}$ & $0(15)$ & $\mathbf{0}$ & 100 & $0(1)$ & $100(1)$ & $100(1)$ & $75(12)$ & $\mathbf{0}$ & $0(2)$ & $\mathbf{0}$ & 0 \\
\hline $\begin{array}{l}\text { light) } \\
\text { Utilization as sole }\end{array}$ & & & & & & & & & & & \\
\hline Acetate & 40(15) & 0 & 0 & 0 & $\mathbf{0}$ & 67 & 75 & 0 & 14 & $\mathbf{0}$ & 0 \\
\hline Beta-alanine & & ND & 0 & 0 & 0 & 0 & $0(4)$ & ND & $0(4)$ & ND & 0 \\
\hline DL-Alanine & 100 & 100 & 100 & 100 & 50 & 100 & 100 & 100 & 100 & 67 & 100 \\
\hline Alginate & 94 & 0 & 100 & ND & $\mathbf{0}$ & ND & $17(12)$ & 0 & $0(3)$ & 0 & ND \\
\hline Arginine & 94 & 100 & 100 & 100 & 100 & 83 & 94 & 100 & 86 & 0 & 100 \\
\hline Citrate & 94 & 50 & $100(4)$ & 100 & 100 & 100 & 94 & 100 & 100 & 33 & 100 \\
\hline Formate & 0 & 0 & 0 & 0 & 0 & 0 & 0 & 0 & 0 & 0 & 0 \\
\hline Glycine & $40(15)$ & 50 & 0 & 0 & 0 & 17 & 0 & 0 & 0 & 0 & 0 \\
\hline Inulin & 88 & 100 & $100(4)$ & 50 & 100 & 83 & 93(15) & 67 & 86 & 0 & 100 \\
\hline Lysine & $50(14)$ & 0 & 20 & 0 & 0 & 0 & 0 & 0 & 0 & 0 & 0 \\
\hline Malona & 0 & 0 & 0 & 0 & 0 & 0 & 0 & 0 & 0 & 0 & 0 \\
\hline Phenylalanine & 13 & 100 & 20 & 0 & 0 & 17 & 0 & 0 & 0 & $\mathbf{0}$ & 0 \\
\hline Proline & 100 & 50 & 100 & 50 & 100 & 83 & 94 & 100 & 43 & 33 & 100 \\
\hline Propanol & 88 & 0 & 0 & 0 & 0 & 0 & 0 & 0 & 0 & 0 & 0 \\
\hline Pyruvate & $100(15)$ & 100 & 100 & 100 & 100 & 100 & 88 & 33 & 57 & 0 & 100 \\
\hline Serine & 81 & 100 & $25(4)$ & 100 & $0(1)$ & 100 & 100 & 0 & 57 & 0 & 100 \\
\hline Succinate & 93(15) & $0(1)$ & 60 & 100 & 100 & 100 & 63 & 0 & 71 & 0 & 100 \\
\hline Tartrate & $15(13)$ & 0 & $0(4)$ & 0 & 0 & 0 & 0 & 0 & 0 & 0 & 0 \\
\hline Tryptophan & $70(10)$ & 0 & ND & 0 & 0 & 0 & 0 & 0 & 14 & 0 & $0(1)$ \\
\hline Uracil & 0 & 0 & 0 & 0 & 0 & 0 & 0 & 0 & 0 & 0 & 0 \\
\hline
\end{tabular}

a The numbers in parentheses indicate the numbers of strains tested for different traits.

${ }^{b} \mathrm{ND}$, Not done.

$V$. parahaemolyticus, with the exception of sucrose fermentation, which was positive for these strains. Other characteristics that are useful for distinguishing $V$. parahaemolyticus from Vibrio alginolyticus (viz., acetoin production, growth in $10 \% \mathrm{NaCl}$, swarming on $1.5 \%$ agar plates, and methyl red reaction) were found to be typical of $V$. parahaemolyticus $(75)$. Sucrose fermentation was tested with several media, including HughLeifson medium (47), OF basal medium, Leifson MOF medium (63), TSI agar, and TCBS agar. All methods gave the same results (i.e., positive sucrose fermentation).

Two other noteworthy characteristics demonstrated by strains of cluster 12 were that $81 \%$ of the strains were urease positive and $17 \%$ were indole negative.

Cluster 13 consisted of two reference strains of $V$. parahaemolyticus which clustered at a slightly lower value $(72 \%)$. These strains, strains SAK 4 and ATCC 27969 (= FC 1101), gave the biochemical reaction patterns expected for $V$. parahaemolyticus and differed from cluster 12 in being sucrose negative, unable to grow at $44^{\circ} \mathrm{C}$, and unable to utilize alginate, propanol, or succinate as a sole carbon source.

Five shrimp isolates comprised cluster 14 . This cluster was very similar to clusters 12 and 13 and was also identified as $V$. parahaemolyticus. The strains of cluster 14 also were sucrose positive, but possessed none of the other key characteristics of $V$. alginolyticus. These strains differed from the strains of cluster 12 by being urease negative, unable to grow on SS agar, variable for the lysine decarboxylase reaction, and esculin positive.

Phenon 15 comprised only two strains, strains F580/75 and 715. Both of these strains were from Denmark; strain F580/75 was isolated from a fish wound, and strain 715 was isolated from salt water. The strains of cluster 15 were closely related to the strains of cluster 1 ( $V$. anguillarum) but were distinguished by the flat, colorless colonies produced, in addition to the lack of acid production from mannitol or dextrin and the lack of growth at $5^{\circ} \mathrm{C}$. Additional characteristics of this cluster are given in Table 4.

Two more strains from Denmark, strain 
F647/75 from a fish infection and strain 274/75 from seawater, comprised cluster 16, which was rather heterogeneous. Strain F647/75 is a Pseudomonas sp. belonging to group $\mathrm{A}$ of Lee et al. (Lee et al., J. Appl. Bacteriol. 45:viii, 1978) and was identified on the basis of its oxidative metabolism, motility, oxidase reaction, arginine dihydrolase, resistance to chloramphenicol, lack of ornithine decarboxylase, and growth on succinate and proline as sole carbon sources. Strain 274/75 satisfied the minimum requirements for assignment to the genus Vibrio, but could not be unequivocally assigned to any species.

Strains of phena 17 through 20 resembled the species described by Lee et al. (60) and named $V$. metschnikovii. These strains were gram-negative, curved or straight, motile, rod-shaped organisms that were facultatively anaerobic and produced acid, but no gas, from glucose, mannitol, and sucrose. They were susceptible to vibriostatic agent 0/129 and negative for ornithine and lysine decarboxylases, arginine dihydrolase, nitrate reduction, and cytochrome oxidase.

The cytochrome oxidase reaction is the major characteristic that distinguishes $V$. metschnikovii from other Vibrio species. The other characteristics given by Lee et al. (60) are in agreement with our results (i.e., production of acid from mannose and trehalose and no acid produced from arabinose, raffinose, rhamnose, salicin, or xylose). Our results and those of Lee et al. also agreed with respect to utilization of sole carbon sources; nearly all strains were unable to utilize formate, glycine, lysine, malonate, phenylalanine, propanol, or tartrate, whereas they were able to utilize alanine and proline. Variable reactions were observed in the arginine, pyruvate, serine, and succinate tests. In addition, almost all strains hydrolyzed starch, chitin, gelatin, and casein and produced deoxyribonuclease and lipase (Tween 80).

The largest of the $V$. metschnikovii clusters was cluster 18; this cluster consisted of 16 strains, all of which were environmental isolates from Denmark. Three more isolates from Denmark were recovered in cluster 19 , which joined cluster 18 at the $71 \%$ level. These strains were distinguished from the cluster 18 strains and other $V$. metschnikovii strains by not producing acid from galactose, inositol, lactose, and melibiose and by not being able to utilize succinate as a sole carbon source or hydrolyze Tween 60 , although all strains could hydrolyze Tween 80 .

Phenon 20 is a very interesting group, consisting entirely of reference strains of $V$. metschnikovii and Vibrio proteus. V. proteus is an illegitimate name and is a synonym for $V$. metschnikovii (60). The strains in this group included strain 10041-74 (V. proteus NCTC 8563), strain 1004074 (V. metschnikovii 7), strain 10122-74 ( $V$. metschnikovii ATCC 7708), strain 10121-74 ( $V$. metschnikovii 211), strain 10042-74 (V. metschnikovii NCTC 8443), strain 10036-74 (V. metschnikovii CIP 6914), and strain 8394-62 (V. cholerae $0-7$ ). These reference strains clustered with the wild strains at similarity values of only $\geq 66 \%$; this finding was similar to the finding of Lee et al. (60), who reported that all of the reference strains of both $V$. proteus and $V$. metschnikovii clustered separately from the wild strains. The strains which we used behaved exactly like the strains in the previous study in that all strains except one were unable to grow on TCBS agar, the primary isolation medium used for Vibrio species, and none could grow on SS agar. All of the wild strains grew well on these media. Table 4 shows that, in addition to not growing on TCBS agar, most of the phenon 20 strains did not grow on agar containing sodium lauryl sulfate or brilliant green. All strains produced unusually long cells, with ratios of length to width of $>6: 1$. One notable biochemical reaction was the production of acid from inositol by all of these strains, a characteristic negative or variable for the other clusters of $V$. metschnikovii strains. The phenon 20 strains were not as nutritionally diverse as the wild strains; the percent carbon source utilization was generally lower for these strains as a cluster than for the strains in the other clusters of $V$. metschnikovii.

Cluster 21 consisted of three strains (strains $639 / 75,1106 / 75$, and 1211/75), all from Denmark. These strains were closely related to the strains of phenon 2; in addition, their characteristics were very similar to those of $V$. anguillarum and, therefore, they were identified as $V$. anguillarum. These strains differed from those of phenon 2 by not being able to utilize citrate or proline (Table 5).

Two more of the Danish strains were grouped in phenon 22; these were strain F702/75, which was originally isolated from a fish infection, and strain DAM81/75, which was isolated from Copenhagen sewage waters. This cluster had an intergroup similarity value of $70 \%$ compared with phenon 5 , which included strains of $V$. cholerae from Copenhagen sewage water. We concluded that strain DAM81/75 is a strain of $V$. cholerae; this strain had a mean similarity value of $76 \%$ compared with group 5 . When clustering was performed by single linkage rather than average linkage, strain DAM81/75 clustered with phenon 5, having a similarity value of $81 \%$ compared with strain DAM86/75 in phenon 5 (Kaper and Colwell, unpublished data). However, because of lower similarity values in comparisons with other strains of phenon 5 , the average level of similarity was higher with strain F702/75 than with all strains of phenon 5 . The same 
TABLE 5. Characteristics of Vibrio phena 23 through 33

\begin{tabular}{|c|c|c|c|c|c|c|c|c|c|c|c|}
\hline \multirow[b]{2}{*}{ Determination } & \multicolumn{11}{|c|}{$\%$ of strains positive in phenon: } \\
\hline & $\begin{array}{c}23 \\
(n=3)\end{array}$ & $\begin{array}{c}24 \\
(n=2)\end{array}$ & $\begin{array}{c}25 \\
(n=2)\end{array}$ & $\begin{array}{c}26 \\
(n=2)\end{array}$ & $\begin{array}{c}27 \\
(n=2)\end{array}$ & $\begin{array}{c}28 \\
(n=2)\end{array}$ & $\begin{array}{c}29 \\
(n=3)\end{array}$ & $\begin{array}{c}30 \\
(n=6)\end{array}$ & $\begin{array}{c}31 \\
(n=2)\end{array}$ & $\begin{array}{c}32 \\
(n=13)\end{array}$ & $\begin{array}{c}33 \\
(n=2)\end{array}$ \\
\hline \multicolumn{12}{|l|}{ Colonial morpholo- } \\
\hline $\begin{array}{l}\text { gy } \\
\text { White or cream }\end{array}$ & 100 & $0(1)^{a}$ & 0 & 0 & 0 & 0 & 33 & $60(5)$ & 0 & 100 & 100 \\
\hline Yellow & 0 & $0(1)$ & 0 & 0 & 0 & 0 & 0 & $20(5)$ & 0 & 0 & 0 \\
\hline Colorless & $\mathbf{0}$ & $100(1)$ & 50 & 0 & 0 & 100 & 0 & $20(5)$ & 100 & 0 & 0 \\
\hline Diffusible pigment & $\mathbf{0}$ & 0 & 0 & $\mathbf{0}$ & $\mathbf{0}$ & 0 & 0 & 33 & 0 & $\mathbf{0}$ & $O(1)$ \\
\hline Mucoid & 0 & $0(1)$ & 0 & 0 & 0 & 0 & 0 & $0(5)$ & $\mathbf{0}$ & 0 & $\mathbf{0}$ \\
\hline Matt & 0 & 0 & 0 & 0 & 50 & 0 & 0 & 17 & 0 & 31 & $100(1)$ \\
\hline Translucent & 0 & 50 & 100 & 100 & 100 & 100 & 67 & 83 & 50 & 15 & $100(1)$ \\
\hline Round & 100 & 50 & 100 & 100 & 50 & 100 & 100 & 100 & 100 & 69 & $100(1)$ \\
\hline Irregular margin & 0 & 0 & 0 & $\mathbf{0}$ & 50 & 0 & 0 & 0 & 0 & 15 & $0(1)$ \\
\hline Rhizoid margin & 0 & 50 & 0 & 0 & 0 & 0 & 0 & 0 & 0 & 15 & $0(1)$ \\
\hline Flat & 0 & 0 & 50 & 50 & 100 & 0 & 0 & 0 & 0 & 92 & $100(1)$ \\
\hline Spreading & 0 & 0 & 0 & 0 & 0 & 0 & 0 & 0 & 0 & 0 & $0(1)$ \\
\hline \multicolumn{12}{|l|}{ Micromorphology } \\
\hline Gram variable & 0 & 0 & $\mathrm{ND}^{b}$ & $0(1)$ & ND & 50 & 0 & $0(5)$ & $0(1)$ & $50(12)$ & $100(1)$ \\
\hline $\begin{array}{l}\text { Length/width ratio } \\
\text { of } \geq 6: 1\end{array}$ & 33 & 0 & ND & 0 & $0(1)$ & 0 & 0 & $0(5)$ & $100(1)$ & $0(12)$ & $0(1)$ \\
\hline Filaments & 67 & 0 & ND & 0 & 0 & 0 & 0 & $20(5)$ & $0(1)$ & $0(12)$ & $0(1)$ \\
\hline Motility & $100(1)$ & $100(1)$ & 100 & 100 & 0 & $100(1)$ & ND & $100(4)$ & ND & $100(3)$ & $100(1)$ \\
\hline Fluorescence & 0 & 0 & 0 & 0 & 0 & 0 & 0 & 50 & 0 & 0 & 0 \\
\hline \multicolumn{12}{|l|}{ Growth at: } \\
\hline $\begin{array}{r}5^{\circ} \mathrm{C} \\
10^{\circ} \mathrm{C}\end{array}$ & $100(2)$ & 0 & 0 & 0 & 0 & 0 & 100 & 100 & 0 & $0(11)$ & 0 \\
\hline $\begin{array}{l}15^{\circ} \mathrm{C} \\
15^{\circ} \mathrm{C}\end{array}$ & 100 & 100 & ND & $100(1)$ & $100(1)$ & 100 & 100 & 100 & 50 & 85 & 100 \\
\hline $42^{\circ} \mathrm{C}$ & & 100 & 100 & 100 & 100 & 100 & 100 & 100 & 50 & 100 & 100 \\
\hline $\begin{array}{l}42^{\circ} \mathrm{C} \\
44^{\circ} \mathrm{C}\end{array}$ & $0 /$ & 100 & 100 & 100 & 1100 & 100 & 1100 & 67 & 100 & 100 & 100 \\
\hline & 33 & 100 & 100 & 100 & 100 & 0 & 33 & 50 & 100 & $100(12)$ & 100 \\
\hline pF & $100(2)$ & $100(1)$ & 50 & 100 & 100 & 50 & 100 & $100(5)$ & ND & $67(3)$ & ND \\
\hline pH 9.0 & 100 & $100(1)$ & 100 & 100 & 100 & 0 & 100 & 83 & 0 & 100 & 100 \\
\hline pH 9.5 & ND & 100 & ND & ND & ND & ND & ND & 83 & 0 & 100 & 100 \\
\hline \multicolumn{12}{|l|}{ Growth on: } \\
\hline $0 \% \mathrm{NaCl}$ & $0(2)$ & 0 & 100 & 100 & 100 & 0 & 100 & $100(5)$ & $100(1)$ & $100(11)$ & 100 \\
\hline $7 \% \mathrm{NaCl}$ & 0 & 100 & 0 & 0 & 0 & 0 & $50(2)$ & 33 & 100 & 100 & 100 \\
\hline $10 \% \mathrm{NaCl}$ & 0 & 100 & 0 & 0 & 0 & 0 & 0 & 33 & 100 & 92 & 100 \\
\hline $\begin{array}{l}\text { Sodium lauryl } \\
\text { sulfate }\end{array}$ & 100 & 100 & 100 & 100 & 100 & 50 & 100 & 100 & 100 & 39 & 100 \\
\hline Crystal violet & $0(2)$ & 100 & 50 & 50 & 100 & $0(1)$ & 100 & 100 & 100 & $100(12)$ & 100 \\
\hline Brilliant green & 33 & 100 & 0 & 100 & 0 & 0 & 100 & 100 & 100 & 92 & $100(1)$ \\
\hline TCBS agar & 100 & 100 & ND & 50 & 50 & 100 & $100(1)$ & 100 & 0 & 100 & 100 \\
\hline SS agar & 33 & 100 & 100 & 100 & 100 & 0 & 100 & 100 & 0 & 0 & 0 \\
\hline \multicolumn{12}{|l|}{ Resistant to: } \\
\hline Gentamicin & 0 & 0 & 0 & 0 & 0 & 0 & 0 & $0(4)$ & ND & 0 & $0(1)$ \\
\hline Penicillin & 100 & 50 & 100 & 100 & 100 & 100 & 100 & $25(4)$ & ND & 0 & $0(1)$ \\
\hline Chloramphenicol & 0 & 0 & 50 & 0 & $\mathbf{0}$ & 0 & 100 & $0(4)$ & ND & 8 & $0(1)$ \\
\hline Erythromycin & 0 & 0 & 0 & 50 & 50 & 0 & 100 & $33(3)$ & ND & $0(12)$ & $0(1)$ \\
\hline Kanamycin & 0 & 0 & 0 & $\mathbf{0}$ & 0 & 0 & 0 & $0(4)$ & ND & 0 & $0(1)$ \\
\hline Chloramphenicol & ND & 0 & ND & ND & ND & ND & ND & $0(4)$ & ND & 0 & $0(1)$ \\
\hline Novobiocin & 0 & 0 & 0 & 0 & 100 & 0 & 100 & $0(4)$ & ND & 0 & $0(1)$ \\
\hline Polymyxin B & 33 & 100 & 0 & 100 & 100 & 50 & 100 & $75(4)$ & ND & 69 & $0(1)$ \\
\hline Oxytetracycline & ND & 0 & 0 & 0 & 50 & 0 & 33 & $0(4)$ & ND & 0 & $0(1)$ \\
\hline Tetracycline & 0 & 50 & 0 & 0 & 0 & 0 & 0 & $0(4)$ & ND & 0 & $0(1)$ \\
\hline Ampicillin & 0 & 50 & 50 & 0 & 0 & 50 & 100 & $50(4)$ & ND & 0 & $O(1)$ \\
\hline Streptomycin & ND & 0 & ND & ND & ND & ND & ND & $0(4)$ & ND & 0 & $0(1)$ \\
\hline $\begin{array}{l}\text { Susceptible to vi- } \\
\text { briostatic agent } 0 / \\
129\end{array}$ & 100 & $0(1)$ & 100 & 0 & 0 & $100(1)$ & 0 & 33 & ND & 100 & 0 \\
\hline \multicolumn{12}{|l|}{ Biochemical tests } \\
\hline Catalase & 100 & 100 & 100 & 100 & 100 & 50 & 100 & 100 & 100 & 100 & $100(1)$ \\
\hline Oxidase & 100 & 100 & 100 & 50 & 0 & 100 & 100 & 100 & 100 & $0(12)$ & $0(1)$ \\
\hline $\begin{array}{l}\text { Arginine dihydro- } \\
\text { lase }\end{array}$ & 0 & 100 & 100 & 100 & 100 & 0 & 100 & 67 & ND & 0 & $0(1)$ \\
\hline $\begin{array}{l}\text { Histidine decar- } \\
\text { boxylase }\end{array}$ & $\mathbf{0}$ & 0 & 100 & 50 & 50 & $\mathbf{0}$ & 0 & $\mathbf{0}$ & $0(1)$ & 0 & $0(1)$ \\
\hline
\end{tabular}


TABLE 5-Continued

\begin{tabular}{|c|c|c|c|c|c|c|c|c|c|c|c|}
\hline \multirow[b]{2}{*}{ Determination } & \multicolumn{11}{|c|}{$\%$ of strains positive in phenon: } \\
\hline & $\begin{array}{c}23 \\
(n=3)\end{array}$ & $\begin{array}{c}24 \\
(n=2) \\
\end{array}$ & $\begin{array}{c}25 \\
(n=2)\end{array}$ & $\begin{array}{c}26 \\
(n=2)\end{array}$ & $\begin{array}{c}27 \\
(n=2)\end{array}$ & $\begin{array}{c}28 \\
(n=2)\end{array}$ & $\begin{array}{c}29 \\
(n=3)\end{array}$ & $\begin{array}{c}30 \\
(n=6)\end{array}$ & $\begin{array}{c}31 \\
(n=2)\end{array}$ & $\begin{array}{c}32 \\
(n=13)\end{array}$ & $\begin{array}{c}33 \\
(n=2)\end{array}$ \\
\hline $\begin{array}{l}\text { Lysine decarbox- } \\
\text { ylase }\end{array}$ & $\mathbf{0}$ & 100 & 100 & 50 & $\mathbf{0}$ & 50 & $\mathbf{0}$ & $\mathbf{0}$ & $0(1)$ & o(11) & 0 \\
\hline $\begin{array}{l}\text { Ornithine decar- } \\
\text { boxylase }\end{array}$ & 0 & 100 & 100 & 50 & 50 & 0 & $\mathbf{0}$ & $\mathbf{0}$ & ND & 8 & 0 \\
\hline $\mathrm{H}_{2} \mathrm{~S}$ & $\mathbf{0}$ & $\mathbf{0}$ & $\mathbf{0}$ & $\mathbf{0}$ & 0 & 0 & 0 & 0 & 0 & 0 & $\mathbf{0}$ \\
\hline Methyl red & $\mathbf{0}$ & 50 & 100 & 100 & 100 & 100 & 0 & $\mathbf{0}$ & 50 & 23 & $\mathbf{0}$ \\
\hline Voges-Proskauer & $100(1)$ & $\mathbf{0}$ & $\mathbf{0}$ & 0 & 50 & $0(1)$ & ND & 67 & 0 & 92 & 50 \\
\hline $\begin{array}{l}\text { Phenylalanine de- } \\
\text { aminase }\end{array}$ & 0 & $\mathbf{0}$ & $\mathbf{0}$ & 0 & $\mathbf{0}$ & 0 & 0 & 0 & $0(1)$ & 0 & 0 \\
\hline Nitrate reduction & 100 & 100 & 100 & 100 & 100 & 100 & ND & 0 & 100 & $\mathbf{0}$ & 0 \\
\hline Nitrite reduction & ND & 100 & ND & ND & ND & ND & ND & $\mathbf{0}$ & 50 & $\mathbf{0}$ & 0 \\
\hline Phosphatase & $0(1)$ & 100 & $100(1)$ & 100 & 100 & 100 & 100 & 100 & 100 & 31 & 100 \\
\hline Indole & $67^{-1}$ & 0 & 100 & 100 & 0 & 0 & 0 & 0 & ND & 0 & 0 \\
\hline Levan & $\mathbf{0}$ & $\mathbf{0}$ & $\mathbf{0}$ & $\mathbf{0}$ & 0 & $\mathbf{0}$ & 0 & $0(5)$ & 0 & 0 & 0 \\
\hline Melanin & $\mathbf{0}$ & $\mathbf{0}$ & $0(1)$ & $\mathbf{0}$ & $0(1)$ & $\mathbf{0}$ & $\mathbf{0}$ & 0 & 0 & 0 & 0 \\
\hline $\begin{array}{l}\text { Iodacetate, } \\
\text { growth }\end{array}$ & $\mathbf{0}$ & 100 & $\mathbf{0}$ & $\mathbf{0}$ & $\mathbf{0}$ & $\mathbf{0}$ & 100 & 83 & $0(1)$ & $75(8)$ & $\mathbf{O ( 1 )}$ \\
\hline Iodacetate, acid & $\mathbf{0}$ & $\mathbf{0}$ & 0 & 0 & 0 & $\mathbf{0}$ & 0 & 67 & $0(1)$ & $0(8)$ & $0(1)$ \\
\hline Alpha-hemolysis & $\mathbf{0}$ & $\mathbf{0}$ & 0 & $\mathbf{0}$ & $\mathbf{0}$ & 0 & 0 & 0 & 0 & $0(11)$ & 50 \\
\hline Beta-hemolysis & 100 & $\mathbf{0}$ & $\mathbf{0}$ & 0 & 0 & 0 & 67 & 67 & 0 & $73(11)$ & 50 \\
\hline Simmons citrate & ND & $100(1)$ & ND & $\mathbf{0}$ & $\mathbf{0}$ & 0 & ND & $80(5)$ & ND & $33(3)$ & ND \\
\hline $\begin{array}{l}\text { Ammonia produc- } \\
\text { tion }\end{array}$ & 100 & ND & $\mathbf{0}$ & 50 & 100 & 100 & 100 & ND & ND & ND & ND \\
\hline $\begin{array}{l}\text { Fermentative me- } \\
\text { tabolism }\end{array}$ & 100 & $100(1)$ & 100 & 100 & 100 & $100(1)$ & $\mathbf{0}$ & 0 & 50 & $8(12)$ & 0 \\
\hline Acid fron & & & & & & & & & & & \\
\hline & 67 & 100 & $\mathbf{0}$ & $\mathbf{0}$ & $\mathbf{0}$ & $\mathbf{0}$ & 100 & 100 & $\mathbf{0}$ & 0 & $\mathbf{0}$ \\
\hline Butanol & 0 & 50 & $\mathbf{0}$ & 0 & 0 & $\mathbf{0}$ & $\mathbf{0}$ & 50 & $\mathbf{0}$ & $\mathbf{0}$ & $\mathbf{0}$ \\
\hline Cellobiose & 100 & 50 & 0 & 0 & 0 & $100(1)$ & 33 & 100 & 0 & $67(12)$ & 50 \\
\hline Dextrin & $\mathbf{0}$ & 100 & $\mathbf{0}$ & 0 & 0 & 0 & 0 & $\mathbf{0}$ & 50 & 0 & $\mathbf{0}$ \\
\hline Erythritol & 0 & 0 & 0 & 0 & 0 & 0 & 100 & 0 & $\mathbf{0}$ & $\mathbf{0}$ & 0 \\
\hline Ethanol & $\mathbf{0}$ & 100 & $\mathbf{0}$ & $\mathbf{0}$ & $\mathbf{0}$ & $\mathbf{0}$ & 67 & 50 & $\mathbf{0}$ & 0 & $\mathbf{0}$ \\
\hline Fructose & 100 & 100 & 0 & 50 & $100(1)$ & 100 & 100 & 100 & 100 & 23 & 0 \\
\hline Galactose & 100 & 100 & 100 & $\mathbf{0}$ & 50 & $\mathbf{0}$ & 100 & 100 & $\mathbf{0}$ & 0 & 0 \\
\hline Glycero & 100 & 100 & 100 & 0 & 0 & 0 & 100 & 33 & 50 & 61 & 50 \\
\hline Inosit & $\mathbf{0}$ & $\mathbf{0}$ & 100 & 50 & $\mathbf{0}$ & $\mathbf{0}$ & 0 & $40(5)$ & $\mathbf{0}$ & 0 & 0 \\
\hline Lactos & 0 & 0 & 100 & 0 & $\mathbf{0}$ & 0 & $0(2)$ & 0 & $\mathbf{0}$ & $0(12)$ & 0 \\
\hline Mannitol & 100 & 100 & 0 & 0 & 0 & $100(1)$ & 100 & 100 & 50 & $67(12)$ & 100 \\
\hline Mannos & 67 & 100 & 0 & 50 & 0 & 50 & 100 & 100 & 0 & 100 & 50 \\
\hline Meli & 0 & 0 & 100 & 0 & 0 & 0 & 100 & 100 & 0 & 0 & 0 \\
\hline Raffinos & o & 0 & 0 & 0 & 0 & 0 & 0 & 0 & 0 & 0 & 0 \\
\hline Rhamnos & o & 0 & 0 & 0 & 0 & 50 & 100 & 100 & 0 & 8 & 0 \\
\hline Salici & 0 & 0 & $0(1)$ & $0(1)$ & 0 & 50 & 0 & 0 & 0 & $75(12)$ & 50 \\
\hline Sorbito & 100 & 0 & 0 & 0 & 0 & 0 & 100 & 83 & 0 & 0 & 0 \\
\hline Sorbo & 0 & 0 & 0 & 0 & 0 & 0 & 0 & 0 & 0 & 0 & 0 \\
\hline Sucro & ND & 0 & ND & 0 & 50 & $100(1)$ & ND & 17 & 100 & 8 & 0 \\
\hline Trehalose & 100 & 100 & 100 & $100(1)$ & 0 & 0 & 0 & 17 & 0 & 0 & 0 \\
\hline Xylos & $\mathbf{0}$ & $\mathbf{0}$ & 0 & 0 & 0 & 0 & 100 & 100 & 0 & 0 & 0 \\
\hline Gas $f$ & & & & & & & & & & & \\
\hline & 0 & $\mathbf{0}$ & $\mathbf{0}$ & 0 & 0 & 0 & 0 & 0 & $0(1)$ & $0(12)$ & $0(1)$ \\
\hline Lactose & 0 & 0 & $\mathbf{0}$ & 0 & 0 & $\mathbf{0}$ & 0 & $0(5)$ & o & 0 & 0 \\
\hline Degradation o & & & & & & & & & & & \\
\hline Ure & 0 & 0 & $\mathbf{0}$ & $\mathbf{0}$ & 0 & 0 & $0(2)$ & 0 & $0(1)$ & 0 & $\mathbf{0}$ \\
\hline & $50(2)$ & 100 & $\mathbf{0}$ & 0 & $0(1)$ & 100 & $50(2)$ & $0(5)$ & 100 & 0 & $\mathbf{0}$ \\
\hline Chitin & ND & 100 & ND & 0 & $0(1)$ & ND & ND & $O(5)$ & ND & $0(12)$ & $0(1)$ \\
\hline Gelatin & ND & $100(1)$ & ND & 0 & 0 & ND & ND & 83 & 50 & 100 & 100 \\
\hline Casein & $100(2)$ & 0 & 100 & 100 & 100 & o & 67 & 100 & o & 100 & 50 \\
\hline DNA & ND & 100 & ND & 0 & 0 & $100(1)$ & ND & $O(5)$ & $O(1)$ & 46 & 0 \\
\hline Tween 20 & 100 & 100 & 100 & 100 & 100 & 100 & 100 & 100 & 100 & $33(12)$ & 100 \\
\hline Tween 40 & 100 & 50 & 100 & 100 & 100 & 100 & 100 & $100(5)$ & 100 & $22(9)$ & $100(1)$ \\
\hline Tween 60 & 100 & 50 & 100 & 100 & 100 & 100 & 100 & 100 & 100 & 15 & 50 \\
\hline Tween 80 & 100 & 100 & 100 & 100 & 100 & 100 & 100 & 100 & o & 100 & 100 \\
\hline Lecithin & $100(2)$ & 50 & $O(1)$ & 0 & 0 & ND & $0(2)$ & $0(5)$ & 0 & $100(11)$ & 100 \\
\hline
\end{tabular}


TABLE 5-Continued

\begin{tabular}{|c|c|c|c|c|c|c|c|c|c|c|c|}
\hline \multirow[b]{2}{*}{ Determination } & \multicolumn{11}{|c|}{$\%$ of strains positive in phenon: } \\
\hline & $\begin{array}{c}23 \\
(n=3)\end{array}$ & $\begin{array}{c}24 \\
(n=2)\end{array}$ & $\begin{array}{c}25 \\
(n=2)\end{array}$ & $\begin{array}{c}26 \\
(n=2)\end{array}$ & $\begin{array}{c}27 \\
(n=2)\end{array}$ & $\begin{array}{c}28 \\
(n=2)\end{array}$ & $\begin{array}{c}29 \\
(n=3)\end{array}$ & $\begin{array}{c}30 \\
(n=6)\end{array}$ & $\begin{array}{c}31 \\
(n=2) \\
\end{array}$ & $(n=13)$ & $\begin{array}{c}33 \\
(n=2)\end{array}$ \\
\hline Tyrosine & 0 & $\mathbf{0}$ & $0(1)$ & 0 & 0 & 0 & 100 & 83 & 0 & 0 & 0 \\
\hline Allantoin & $\mathbf{0}$ & ND & 50 & 100 & 100 & $\mathbf{0}$ & 0 & ND & ND & ND & ND \\
\hline Chondroitin & ND & $0(1)$ & ND & ND & ND & ND & ND & $20(5)$ & 0 & $10(10)$ & 0 \\
\hline Esculin (by color) & ND & 100 & 0 & 0 & 0 & 100 & 0 & 33 & $\mathbf{0}$ & 77 & $100(1)$ \\
\hline $\begin{array}{l}\text { Esculin (by UV } \\
\text { light) }\end{array}$ & ND & $0(1)$ & 0 & $\mathbf{0}$ & 0 & ND & $\mathbf{0}$ & $0(5)$ & $\mathbf{0}$ & 77 & $100(1)$ \\
\hline \multicolumn{12}{|l|}{$\begin{array}{l}\text { Utilization as sole } \\
\text { carbon source: }\end{array}$} \\
\hline Acetate & 0 & 50 & 0 & 100 & 0 & 0 & 67 & 33 & 100 & $25(12)$ & 100 \\
\hline Beta-alanine & 0 & 0 & 0 & $\mathbf{0}$ & 0 & 0 & 0 & $100(5)$ & 100 & $0(9)$ & 50 \\
\hline DL-Alanine & 67 & 100 & 100 & 100 & 100 & 100 & 100 & 100 & 100 & 100 & 100 \\
\hline Alginate & ND & 50 & ND & ND & ND & ND & ND & 100 & 100 & 15 & 0 \\
\hline Arginine & 33 & 100 & 100 & 100 & 100 & 50 & 67 & 100 & 100 & 100 & 50 \\
\hline Citrate & 100 & 100 & 100 & 100 & 50 & 100 & 100 & $100(5)$ & 100 & $100(12)$ & 100 \\
\hline Formate & 0 & 0 & 0 & 0 & 0 & 0 & 67 & 67 & 0 & 0 & 0 \\
\hline Glycine & 0 & 100 & 0 & 0 & 0 & 0 & 0 & 17 & $\mathbf{0}$ & 8 & 100 \\
\hline Inulin & 67 & 100 & 100 & 100 & 100 & 100 & 67 & 100 & 100 & 100 & 100 \\
\hline Lysine & 0 & 0 & 0 & 0 & 0 & 0 & 0 & 100 & 100 & $0(12)$ & 0 \\
\hline Malonate & 0 & 0 & 0 & 0 & 0 & 0 & 0 & 50 & 100 & 0 & 100 \\
\hline Phenylalanine & 0 & 100 & 0 & 0 & 0 & 0 & 0 & 100 & 100 & 0 & 100 \\
\hline Proline & 67 & 100 & 50 & 0 & 100 & 0 & 33 & 100 & 100 & 100 & 100 \\
\hline Propanol & 0 & 100 & 0 & 0 & 0 & 0 & 0 & 100 & 100 & 0 & 100 \\
\hline Pyruvate & 100 & 100 & 100 & 100 & 100 & 100 & 100 & 100 & 100 & 100 & 100 \\
\hline Serine & 67 & 100 & 100 & 100 & 100 & 100 & 100 & 100 & 50 & $42(12)$ & 100 \\
\hline Succinate & 67 & 100 & 100 & 100 & 100 & 100 & 67 & 83 & 100 & 100 & 100 \\
\hline Tartrate & 0 & 50 & 0 & 0 & 0 & 0 & 0 & 100 & 100 & $0(12)$ & 100 \\
\hline Tryptophan & 0 & 100 & 0 & 0 & 0 & 0 & 0 & 100 & 100 & 0 & 50 \\
\hline Uracil & 0 & 0 & 0 & 0 & 0 & 0 & 0 & 17 & 0 & 0 & 0 \\
\hline
\end{tabular}

${ }^{a}$ The numbers in parentheses indicate the numbers of strains tested for different traits.

${ }^{b}$ ND, Not done.

situation exists for strain F702/75, which had a similarity value of $80 \%$ compared with $V$. anguillarum strain F706/75, but lower similarity values compared with other strains of phenon 1 . Strain F702/75 was assigned to phenon 1 by single linkage but not on the basis of average linkage. Thus, cluster 22 consists of strains of two species, $V$. cholerae (strain DAM81/75) and $V$. anguillarum (strain F702/75); this is apparently an aberrancy arising from the limitations of two-dimensional cluster analysis, when in fact multidimensional relationships are involved.

Three strains isolated from fish wounds were recovered in cluster 23 . We concluded that strains F406/75, F407/75, and F678/75 are Vibrio sp.; these organisms were gram negative, facultatively anaerobic, motile, rod-shaped, oxidase positive, and susceptible to vibriostatic agent $0 / 129$. They did not grow in 0 or $7 \% \mathrm{NaCl}$, produced acid but no gas from glucose, cellobiose, galactose, glycerol, mannitol, and trehalose, and reduced nitrate to nitrites, in addition to being lipolytic and proteolytic (Table 5). Decarboxylase reactions were negative for all amino acids tested. Assignment to a described spe- cies of the genus Vibrio was difficult. We concluded that these strains comprise a new species.

Phenon 24 consists of two strains (strains K19 and KALV 18), both of which were isolated from seawater in Denmark. These strains were motile, facultatively anaerobic, rod-shaped organisms which did not grow in $0 \% \mathrm{NaCl}$ or at $5^{\circ} \mathrm{C}$, but did grow at all other salinities and temperatures tested. They were resistant to vibriostatic agent $0 / 129$ at concentrations of 100 and $150 \mu \mathrm{g} / \mathrm{ml}$ but not at higher concentrations (e.g., $300 \mu \mathrm{g} / \mathrm{ml}$ ), characteristic of Aeromonas spp. They were extremely versatile, both physiologically and biochemically, and utilized all sole carbon sources tested except $\beta$-alanine, formate, lysine, malonate, and uracil (Table 5). These strains could not be classified as Vibrio, Beneckea, or Aeromonas and did not fall into any other genus comprised of facultatively anaerobic rod-shaped organisms. Hence, identification must await further characterization.

Phena 25 through 27 contained reference strains of Plesiomonas and Shigella spp. which were provided by $R$. Sakazaki. None of the 
environmental isolates of vibrios clustered with these reference strains.

Cluster 28 consisted of two strains (strains $1157 / 75$ and 1114/75); both of these strains were from Denmark, and they clustered at the $71 \%$ level of similarity. They were gram-negative, oxidase-positive, motile, facultatively anaerobic, rod-shaped organisms that were halophilic, growing at $3 \% \mathrm{NaCl}$ but not at 0 or $7 \% \mathrm{NaCl}$; they were susceptible to vibriostatic agent $0 / 129$ and produced acid from cellobiose, fructose, mannitol, and sucrose. These strains digested starch but not casein and were lipolytic. Strains $1157 / 75$ and $1114 / 75$ are considered to be representatives of a Vibrio sp., but were intermediate to, rather than belonging in, previously described species of this genus.

Pseudomonas spp. were recovered in phena 29 and 30 , which contained three and six strains, respectively, all of which were isolated in Denmark. These strains possessed the minimal characteristics of the genus Pseudomonas (46). They were gram-negative, aerobic, motile, rod-shaped organisms that did not produce acid from glucose in closed tubes. They were oxidase and catalase positive and negative in the indole and methyl red tests. These strains were identified as Pseudomonas aeruginosa on the basis of a positive arginine dihydrolase test, growth at $42^{\circ} \mathrm{C}$, and production of acid from xylose, mannitol, arabinose, and rhamnose but not from lactose or salicin (Table 5).

Two reference strains of Vibrio marinus grouped together in cluster 31 . No other strains examined in this study clustered with $V$. marinus.

Another Pseudomonas species was found in phenon 32. The 13 strains of phenon 32 were all isolated in Denmark and exhibited key characteristics of the genus Pseudomonas (see above), except that they were oxidase negative, suggesting that these strains are Pseudomonas maltophila. The other characteristics of these strains (Table 5) support this conclusion (viz., motility, production of lipase (Tween 80 ), no starch hydrolysis, gelatin hydrolysis, and production of acid from mannose).

The final cluster in the analysis consisted of two strains, strain 613/75 from Denmark and strain ATCC 25916, which was originally deposited as Beneckea pelagia. This cluster varied in many characteristics from the description of $B$. pelagia by Baumann et al. (6), such as being nonfermentative and oxidase negative (both should be positive). The reason for the discrepancies is not known.

\section{DISCUSSION}

The strains of Vibrio and related organisms examined in this study clustered into 33 phena, the formation of which crossed geographic and specimen boundaries. Conclusions relating to the ecology and taxonomy of these strains are discussed below by species.

$V$. cholerae. A total of 43 strains of $V$. cholerae was examined by numerical taxonomy. The results of this analysis support the conclusions of Colwell (13), Sakazaki et al. (76), Shewan and Veron (82), and other workers in that no taxonomic separation could be made in this species on the basis of serology (specifically, the possession of the $\mathrm{O} 1$ antigen by these strains). Although this classification is not accepted by many medical microbiologists and epidemiologists $(18,30)$, it is consistent with all principles of taxonomy and has been confirmed by DNADNA hybridization $(11,97)$. Recent genetic evidence has demonstrated that some strains of $V$. cholerae non-O1 possess genes encoding cholera toxin, whereas many strains of $V$. cholerae 01 do not possess such genes (54). Thus, the results of a molecular genetic analysis of the cholera toxin genes refute the argument for a species defined by possession of a single antigen. Similarly, in the present study strains of $V$. cholerae possessing the $\mathrm{O} 1$ antigen clustered with $V$. cholerae non-O1 strains.

The biotyping scheme of Bergey's Manual (82) is not supported by our results (i.e., the scheme separating $V$. cholerae into the following four biotypes: cholerae [the so-called classical biotype], El Tor, proteus, and albensis). Classical and El Tor strains were grouped together in the present study and were not separated into biotypes or subclusters. On the other hand, the proteus biotype was grouped relatively distantly from $V$. cholerae (clusters 17 through 20). Unfortunately, strains of biotype albensis were not included in this study; therefore, we can make no comment on this biotype. The conclusions described above were reached by the Subcommittee on the Taxonomy of Vibrios (48), which recommended that the proteus and albensis groups should not be designated biotypes of $V$. cholerae. We hope that the next edition of Bergey's Manual will reflect these recommendations.

The strains of $V$. cholerae used in this study formed a fairly homogeneous group, but slight differences among the strains allowed separation into subclusters 5 through 8 . The most notable feature of this separation was the antibiotic resistance demonstrated by strains of clusters 5 through 7, particularly in the case of the antibiotics ampicillin and tetracycline (tetracyline is the antibiotic of choice in the treatment of cholera). Since the majority of the resistant strains were isolated from Copenhagen sewage water (the DAM strains), it is possible that resistance was transferred from coliform bacteria in the sew- 
age. Tetracycline- and ampicillin-resistant $V$. cholerae strains have recently been isolated in Bangladesh (96), and in vitro transfer of $R$ plasmids from Escherichia coli to $V$. cholerae has been reported by several investigators (21, 69,72 ). Interestingly, strains that acquired a plasmid conferring resistance to chloramphenicol, streptomycin, and sulfonamide grew poorly on TCBS agar at temperatures above $37^{\circ} \mathrm{C}(100)$. Since TCBS agar is the standard recovery medium used for $V$. cholerae, caution must be exercised in the choice of the medium used when one is looking for plasmids in $V$. cholerae.

Another characteristic that distinguished $V$. cholerae strains of cluster 7 was fermentation of lactose. $V$. cholerae is considered to be lactose negative. However, this species is sometimes lactose positive after delayed incubation. Sanyal et al. (78) reported that they isolated strains of $V$. cholerae which were capable of rapid fermentation of lactose. Such strains could be misidentified easily. Lactose-positive Salmonella strains have been reported $(27,52)$, which no doubt would have been discarded as coliforms if salmonellae were sought. The ability to ferment lactose has been shown to be plasmid mediated in Salmonella (52) but not in $V$. cholerae. Incidentally, these strains should not be confused with so-called lactose-positive vibrios originally described by Hollis et al. (43) and redescribed as V. vulnificus (28).

Historically, $V$. cholerae has been divided into biotypes on the basis of sucrose, mannose, and arabinose fermentation reactions. These biotypes have been recognized as Heiberg groups (39). Strains classified as Heiberg groups I, II, and V clustered together by numerical taxonomy in this study. Thus, there seems to be poor reproducibility of groupings of strains based on the Heiberg groups, with the possible exception of the sucrose-negative strains or Heiberg group V. This group was recently reported to be a separate species on the basis of DNADNA hybridization values (G. R. Fanning, B. R. Davis, A. G. Steigerwalt, I. K. Wachsmuth, F. W. Hickman, J. J. Farmer III, and D. J. Brenner, Abstr. Annu. Meet. Am. Soc. Microbiol. 1980, D57, p. 47).

However, our data suggest the sucrose-negative $V$. cholerae-like strains are a diverse group and that the strains that share the $0-1$ antigen are most probably a biovar of $V$. cholerae. Studies in progress (P. A. West and R. R. Colwell, unpublished data) clearly indicate heterogeneity within the taxon called Vibrio mimicus. The Heiberg scheme is further weakened by the fact that the arabinose-positive strains (Heiberg groups III and IV) are now recognized to comprise largely aeromonads (76). Clearly, the Heiberg groups, although useful in the early days of bacteriology, no longer can be relied upon for identification of $V$. cholerae.

The largest cluster, cluster 8 , contained strains of various serotypes isolated from around the world. Interestingly, a subgroup of strains was identified within cluster 8. Strains V11, V15, V25, V20, V26, V24, V19, and V29 clustered tightly and differed from the main body of group $8 \mathrm{~V}$. cholerae strains by their failure to ferment dextrin and sucrose, their negative reaction in the Voges-Proskauer test, and their inability to hydrolyze starch. These strains (all Heiberg group V strains) were isolated from a single sampling of Jones Falls water in October 1976. The sample was taken from a single carboy of water analyzed within $45 \mathrm{~min}$ of collection. The fact that these strains are nearly identical (simple matching coefficients as high as $\mathbf{9 8 \%}$ were found) indicates that these strains may be merely clones of an organism found in Baltimore Harbor of Chesapeake Bay, a situation entirely consistent with the hypothesis that $V$. cholerae is an autochthonous bacterial species in Chesapeake Bay and, therefore, can multiply in the bay.

$V$. parahaemolyticus and $V$. alginolyticus. The taxonomy of $V$. parahaemolyticus has been reviewed by Colwell (14), who traced the succession of names given to this organism, including Pasteurella parahaemolytica, Pseudomonas enteritis, Oceanomonas enteritidis, Oceanomonas parahaemolytica, and $V$. parahaemolyticus (so named by Sakazaki et al. [79] after examination of 1,072 strains of this species). Two biotypes were identified, which Zen-Yoji et al. (101) concluded should be separated into two species. In 1968 this was proposed by Sakazaki, who designated biotype 2 as $V$. alginolyticus (75). The main differences between the biotypes are acetoin production, sucrose fermentation, growth in $10 \% \mathrm{NaCl}$, and swarming on agar plates containing 2 to $7 \% \mathrm{NaCl}$. V. parahaemolyticus is negative for these characteristics, whereas $V$. alginolyticus is positive. Later, investigators reported specific DNA homology values of 56 to $70 \%$; these values were higher than the other interspecies homology values for the genus Vibrio $(1,92)$. In the 8th edition of Bergey's Manual these two taxa were combined as $V$. parahaemolyticus, but the Subcommittee on the Taxonomy of Vibrios of the International Committee on Systematic Bacteriology recognized separation of these two taxa as distinct species (48). The widespread acceptance of $V$. alginolyticus by environmental and medical microbiologists portends the acceptance of this species in microbial systematics.

In the present study, 21 strains were identified as $V$. parahaemolyticus. The variation observed in certain characteristics merits discussion. Su- 
crose fermentation is an important feature and is useful for distinguishing $V$. parahaemolyticus from $V$. alginolyticus, but it is not the only feature which can be used for identification. Most of the shrimp isolates in this study were sucrose-positive $V$. parahaemolyticus strains, which is unusual but not unheard of. Colwell (13) found that $6 \%$ of the strains of this species that she examined were sucrose positive, and Gilmour (33) also found several such strains. Kampelmacher (53) isolated sucrose-positive $V$. parahaemolyticus strains from mussels, and $\mathrm{Fu}$ jino et al. (31) found that $2 \%$ of their marine isolates were sucrose positive. Of 1,484 strains of this species isolated from British coastal waters, $6.9 \%$ were sucrose positive (2). Detection of acid from sucrose fermentation by $V$. parahaemolyticus can vary, according to the composition of the medium and the concentration of sucrose used. Baross (Ph.D. thesis, University of Washington, Seattle, 1972) studied this problem and recommended use of the HughLeifson OF base supplemented with $0.5 \%$ (final concentration) sucrose. Several methods were used in the present study (see above), and all yielded the same results. Many of the strains were examined for the presence of extrachromosomal DNA, and the results which we obtained suggest that sucrose fermentation in these strains is not plasmid mediated (Kaper, unpublished data).

Conversely, Stephen et al. (93) found strains of $V$. alginolyticus which did not ferment sucrose but were otherwise identical to the type strains. Golten and Scheffers (34) described strains intermediate between $V$. parahaemolyticus and $V$. alginolyticus which were methyl red and arabinose positive ( $V$. alginolyticus is negative for both tests), sucrose positive, capable of swarming, and able to grow in $10 \% \mathrm{NaCl}$. We observed other unusual characteristics for several strains of $V$. parahaemolyticus. For example, many strains were positive in the urease test, a test which can vary greatly according to the composition of the medium used and how strongly the medium is buffered. Results of two large studies have been contradictory. Sakazaki et al. (77) reported that $4 \%$ of the strains which they tested were urease positive, whereas Colwell (13) reported that $97 \%$ of her strains were urease positive. Recently, a number of clinical isolates of urease-positive $V$. parahaemolyticus have been reported $(50,59)$. The esculin hydrolysis test, which distinguishes the third subgroup of $V$. parahaemolyticus (cluster 14) from the other two, is another test which may provide different results depending on the method used. $V$. parahaemolyticus is usually thought of as being esculin negative (29), but Fujino and coworkers (32) reported that $99 \%$ of their strains were esculin positive. A common test for this characteristic uses a ferric salt indicator, with a brown to black color indicating esculin hydrolysis. Another method (9) calls for the disappearance of UV fluorescence. In the present study we used both methods and found that several strains were positive if the color reaction was the key feature but negative if the tests were read by the UV light reaction. The UV test tended to be too sensitive; even a small amount of residual esculin fluoresced under UV light and gave a false-negative reaction.

$V$. parahaemolyticus is consistently reported as being negative for production of hydrogen sulfide (28). Sakazaki et al. (77) reported that none of the 1,702 strains which they examined were positive for $\mathrm{H}_{2} \mathrm{~S}$ when TSI or SIM agar was used. Using more sensitive methods, Colwell (13) found that nearly all of the $V$. cholerae and $V$. parahaemolyticus strains which she examined produced $\mathrm{H}_{2} \mathrm{~S}$. Twedt and co-workers (98) found that nearly all of their cultures were positive when they used SIM agar and lead acetate agar (Difco). Indeed, hydrogen sulfide production on Russell triple sugar agar but not on TSI has been suggested as an aid in the identification of this species (51). In the present study we used TSI for determinations of $\mathrm{H}_{2} \mathrm{~S}$ production and, not surprisingly, found no strains of $V$. parahaemolyticus that were positive for $\mathrm{H}_{2} \mathrm{~S}$ in this medium.

$V$. anguillarum and other marine vibrios. $V$. anguillarum has long been implicated as a causative agent of fish disease. Originally described in 1909 , the phenon $V$. anguillarum has been repeatedly subdivided and recombined in the literature since that time. This species is readily separated from $V$. cholerae, $V$. parahaemolyti$c u s$, and $V$. alginolyticus by a number of characteristics, mainly the decarboxylase reactions. Differentiation from Aeromonas species can be problematic, unless the proper tests are run (i.e., salt requirement, susceptibility to vibriostatic agent $0 / 129$, and, especially, DNA base composition). Differentiation from other marine vibrios is still difficult, largely because few marine species have been well characterized.

In 1971, Hendrie et al. (41) proposed combining three Vibrio species ( $V$. anguillarum, Vibrio piscium, and Vibrio ichthyodermis) and retaining the name $V$. anguillarum for the phenon. This recommendation was followed in the 8th edition of Bergey's Manual (82). The description provided in Bergey's Manual is very restrictive, however. Many workers have shown that there are significant biochemical variations in a number of features, including the Voges-Proskauer test, the methyl red test, citrate utilization, and fermentation of lactose, arabinose, and sucrose $(25,64)$. The description of Evelyn (25) accounts 
for these variations and appears to be a more reliable description for the species.

A number of biotyping schemes have been proposed for $V$. anguillarum; the first of these was proposed by Nybelin (68), who described two biotypes (type $\mathrm{A}$, which produced indole and acid from mannitol and sucrose, and type B, which was negative for these characteristics). Smith (85) described a third biotype, biotype C, which produced acid from mannitol and sucrose but did not produce indole. Hastein and Smith (38) examined 163 strains of $V$. anguillarum by using principle component analysis and reported two groups which did not correspond to any of the previously described subgroups. Group 1 of Hastein and Smith was arabinose positive, cellobiose positive, and variable for citrate, lactose, and trehalose, whereas group II was arabinose negative, variable for cellobiose, negative for citrate and lactose, and trehalose positive. $\mathrm{Pa}$ cha and Kiehn (70) studied marine vibrio strains isolated from fish in the Pacific Northwest and identified several of these strains as $V$. anguillarum on the basis of morphological and biochemical characteristics. Variations were observed in indole production, gelatin hydrolysis, citrate utilization, and fermentation of arabinose, sucrose, lactose, and glycerol. No consistency was found in these variations, but most strains belonged to group A (68). A biotyping scheme for $V$. anguillarum was recently proposed by Baumann et al. (4), who defined two biotypes on the basis of DNA-DNA hybridization and noted a number of phenotypic reactions that distinguished the strains, including arginine dihydrolase, Voges-Proskauer reactions, growth at $30^{\circ} \mathrm{C}$, reduction of nitrate, and utilization of several compounds as sole carbon sources, including mannose, trehalose, gluconate, succinate, furmarate, and alpha-ketoglutarate. Biotype I strains were positive for all of these tests, whereas biotype II strains were negative for these tests.

Because of the difficulty in distinguishing these strains phenotypically, DNA homology was used to clarify the situation. Kiehn and Pacha (55) hybridized the same strains which they examined phenotypically and found strong correlations between phenotypic and genotypic analyses in a group of eight strains of marine vibrios that were pathogenic for fish. Three groups were discerned by serology, and these were also distinguished by DNA homology. The first group formed on the basis of DNA homology consisted of strains NCMB $6(=$ ATCC 19264), V-2911, V-2916, 30, and 2.1, which were isolated from cod, finnock, Steelhead trout, and Chinook salmon. Strains isolated from Pacific herring (strains 56 and 60) comprised the second group. These strains were not $V$. anguillarum on the basis of the DNA homology results, but they differed only slightly in biochemical characteristics; viz., they were Voges-Proskauer negative, gelatinase negative, and resistant to dihydrostreptomycin. In addition, strain 60 (which had a $\mathrm{G}+\mathrm{C}$ content of $41 \mathrm{~mol} \%$ ) fermented lactose and was indole negative and sucrose negative; this strain was designated a third homology group.

Anderson and Ordal (1) distinguished five DNA homology groups among the marine vibrios, as follows: (i) $V$. parahaemolyticus; (ii) $V$. alginolyticus; (iii) $V$. anguillarum, containing strains NCMB 6, V-2911, and V-2916; (iv) group 4 , strains of which were isolated from diseased herring, including strain 56 of Pacha and Kiehn (67); (v) group 5, which also consisted of strains that were herring isolates (including strain 60) but were genetically distinct from groups 3 and 4. In contrast to the results of Pacha and Kiehn, the results of Anderson and Ordal showed that serology of these strains had little taxonomic value. Subsequent studies have identified two other homology groups. Ordal (unpublished data) defined a homology group of vibrio strains isolated from shellfish, seawater, and sediments. The members of this group were not pathogenic for salmonid fishes, and the group was designated Ki 72-7. Schiewe et al. (80) examined a group of vibrios described by Harrell et al. (36), which exhibited $83 \%$ within-group homology but only about $60 \%$ homology with $V$. anguillarum $V$ 2911. A notable phenotypic feature of these strains is the consistently negative arginine dihydrolase reaction. This group was designated simply by a strain number (V-1669) by Schiewe et al. (80), but was described as V. ("Beneckea") anguillarum biotype II by Baumann et al. (4).

The results of the present study cannot answer unequivocably any of the questions raised above because strains NCMB 6, V-2911, V1669 , and $\mathrm{Ki} 72-7$ and other representatives of the DNA homology groups were not included in this analysis. However, cluster 4 did resemble group V-1669 of Harrell et al. (36). Nevertheless, our results do demonstrate that the biotyping schemes proposed by Nybelin, Smith, and others are not robust. The strain variability in this study was no greater than that observed by Evelyn (25) and other workers for this species. Thus, the differences among the four groups defined in this study were not sufficiently great to warrant separate biotype status. More recently, Schiewe and Crosa (79) reported results of polynucleotide sequence relationship determinations, showing the discrete nature of $V$. anguillarium biotypes 1 and 2 . Furthermore, biotype 2 strains were found to contain a multicopy plasmid that was either identical or highly conserved and unrelated to the virulence plasmid (pJM1) of $V$. anguillarum biotype 1 .

In general, the source of the strains used in 
this study was wound infections of fish. Even though fish pathogenicity tests were not done, we assumed that strains isolated from wounds, which often were in almost pure culture in the wounds, were pathogenic. There was no obvious correlation between pathogenicity and any of the biochemical characteristics. Indeed, characteristics associated with pathogenicity in fish often appear to be plasmid mediated $(19,79)$. It would be interesting to examine selected Danish strains for DNA-DNA hybridization with representatives of the DNA homology groups cited above (all of which were isolated in North America) to assess geographic variation.

V. marinus-Vibrio fischeri. V. marinus was reisolated by Colwell and Morita in 1964 (15), who also gave an emended description of the species. In 1970, Hendrie and co-workers (40) noted similarities between $V$. marinus and $V$. fischeri (formerly Photobacterium fischeri) and proposed that these organisms be amalgamated under the name $V$. fischeri. This approach was followed in the 8th edition of Bergey's Manual (82). Reichelt and Baumann (73) disagreed with the generic assignment of Hendrie and colleagues (40) and argued that $V$. fischeri should remain in the genus Photobacterium. A later paper by these investigators (74) presented data showing essentially no genetic similarity between ' $P$. fischeri' and other Photobacterium spp. Recently, Baumann et al. (5) proposed that these strains, which are distinguished from the rest of the genus Vibrio by low $\mathrm{G}+\mathrm{C}$ contents ( 39 to $42 \mathrm{~mol} \%$ ), should be separated into three species, V. fischeri, Vibrio logei, and V. marinus, reaffirming the results of Colwell and Morita (15) for $V$. marinus. All of these species except $V$. marinus contain luminescent strains.

Unfortunately, in the present study the relationship between $V$. marinus and $V$. fischeri could not be clarified further. No other strains grouped with $V$. marinus, which is not surprising since the majority of strains used in this study were isolated from estuarine rather than marine environments and the isolation procedures used included methods which would not enhance recovery of psychrophiles. The reference strains of $V$. marinus examined in this study were distantly related to other Vibrio spp., indicating that $V$. marinus is indeed a separate species. In the future, it may be shown that this organism is a member of a new genus.

V. metschnikovii. Anyone with extensive experience in the use of TCBS agar has undoubtedly encountered bacteria which, in pure culture, yield sucrose-positive colonies. Upon further testing, these are found to be oxidase negative and, most often, are quickly discarded as not being a Vibrio sp. In fact, many of these strains may belong to the species $V$. metschnikovii, which was recently redescribed by Lee and colleagues (60). Several of the strains included in the present study were identified as $V$. metschnikovii. These clustered together in groups 17 through 20. Interestingly, the analysis of Lee et al. (60) also yielded four clusters of $V$. metschnikovii strains. These authors concluded that the clusters should be put together as a single species and that this species should also include strains heretofore known as $V$. proteus, the original strain of which was isolated in 1884 from feces of patients suffering from cholera (60). This species was listed as a biotype of $V$. cholerae in the 8th edition of Bergey's Manual (82), even though it differs from other biotypes of $V$. cholerae in a number of important features, such as decarboxylase reactions, salt requirements, oxidase reaction, nitrate reduction, and fermentation of lactose and inositol. DNA-DNA hybridization data revealed only 12 to $22 \%$ homology between this species and $V$. cholerae ATCC 14035 (74).

Despite the fact that this species is oxidase negative and not able to reduce nitrates, we concluded that the strains should be classified in the genus Vibrio, based on overall phenotypic similarity and DNA base composition (45.6 mol\% G+C). West et al. (99) examined the cytochrome distribution in $V$. metschnikovii and other Vibrio spp. and found that V. metschnikovii lacks cytochrome $c$ but shares cytochromes $b, o, d$, and $a$ with other Vibrio spp. These workers hypothesized that the different respiratory system compositions may reflect different evolutionary pathways to suit habitats which are dissimilar in degree of anaerobiosis. The answers to this question and questions concerning the taxonomic relationships of $V$. metschnikovii to the other vibrios will require nucleic acid reassociation studies.

One interesting sidelight of our study concerns two strains of $V$. proteus, strains 10037-74 and 10041-74, which are reported to be subcultures of the same strain (strain NCTC 8563). These strains were received from Sakazaki, who had received them from different investigators. These two cultures of the same strain clustered with different phena; the former clustered with group 11, and the latter clustered with group 20 . A parallel situation is found in the work of Lee and co-workers (60), who examined duplicate cultures of three type strains (the duplicate strains were received from different sources). None of the duplicate strains clustered closely together, as might be expected. One of these strains was strain NCTC 8563, as in our study. Probable explanations include the possibility that the strains were mixed in a laboratory and the possibility that the duplicate cultures simply changed (i.e., lost or gained genetic material upon repeated subculturing).

Another curious result was the presence in 
cluster 20 of all of the reference strains which have been in culture collections for several years. These strains utilized fewer organic compounds and were more susceptible to agents such as dyes and detergents than recently isolated environmental isolates. In fact, these strains were not able to grow on TCBS agar, the primary Vibrio isolation medium, whereas fresh isolates readily grew on this medium. This phenomenon was also reported by Lee et al. (60), who observed that all of their reference strains clustered separately from fresh isolates. A possible explanation for this observation is that strains which are held in a culture collection and, particularly in this case, transferred many times change (viz., they grow less rapidly, are less nutritionally versatile, and are highly adapted) to the point of being unable to grow on selective media, such as TCBS agar. Such a situation has been reported for strains of $V$. cholerae (60).

There are a number of species of Vibrio-like organisms which have been described that were not examined in this study. Some species, such as $V$. vulnificus (formerly lactose-positive vibrios) $(8,28)$ and $V$. fluvialis (synonym, group $\mathrm{F}$ vibrios, group EF6) $(49,62 ;$ J. V. Lee, P. Shread, and A. L. Furniss, J. Appl. Bacteriol. 45:ix, 1978) have recently been recognized as human enteric pathogens. Other species, such as Vibrio nigrapulchritudo (7) and Vibrio gazogenes (37), are distinguished chiefly by production of pigment (black and red pigments, respectively). Together, these and similar strains represent a rather diverse group of organisms covering a wide range of salt requirements ( 5 to $700 \mathrm{nM} \mathrm{Na}{ }^{+}$), DNA base compositions (39 to 51 mol\% $\mathrm{G}+\mathrm{C}$ ), DNA homology groups, and ecological niches.

Several genera (Beneckea, Lucibacterium, Oceanomonas, and others) have been proposed to accommodate some of these species. Recently, Baumann et al. (5) proposed that 20 such species should be combined in the genus Vibrio, since no consistent internal subdivisions suitable for generic separation are evident in this complex of strains. We support this concept, having doubted the validity of the above-listed three genera, and offer evidence in the present study, in which strains from a variety of geographic areas, ecological niches, and specimen types were grouped together on the basis of numerical taxonomy. Nevertheless, some species of the genus Vibrio which we examined were quite distantly related to other species in this genus, but reproducible subdivisions could not be discerned. Thus, the internal diversity of this genus is far greater than that of a typical genus of the Enterobacteriaceae. Few other bacterial phena are subdivided and split to the extent of the
Enterobacteriaceae. There is no compelling set of arguments to apply such a degree of division to the genus Vibrio. With our present state of knowledge of the genus Vibrio, subdivisions would be arbitrary, confusing, and, in our opinion, of little value to microbiologists who must deal with these species in clinical and environmental laboratories. For these reasons, we will await the results of experiments in progress in our laboratory before proposing new genera for organisms presently classified as Vibrio spp., regardless of the extent of internal diversity in the genus Vibrio observed in this study.

\section{ACKNOWLEDGMENTS}

This investigation was supported by Public Health Service grant R22-AI-14242 from the National Institutes of Health and by grant DEB-77-14646 from the National Science Foundation. Computer time was made available by the Computer Science Center, University of Maryland, College Park.

\section{LITERATURE CITED}

1. Anderson, R. S., and E. J. Ordal. 1972. Deoxyribonucleic acid relationships among marine vibrios. J. Bacteriol. 109:696-706.

2. Ayres, P. A., and G. I. Barrow. 1978. The distribution of Vibrio parahaemolyticus in British coastal waters: report of a collaborative study, 1975-1976. J. Hyg. 80:281-294.

3. Baird-Parker, A. C. 1963 . A classification of micrococci and staphylococci based on physiological and biochemical tests. J. Gen. Microbiol. 30:409-427.

4. Baumann, P., S. S. Bang, and L. Baumann. 1978. Phenotypic characterization of Beneckea anguillara biotypes I and II. Curr. Microbiol. 1:85-88.

5. Baumann, P., L. Baumann, S. S. Bang, and M. J. Woolkalis. 1980. Reevaluation of the taxonomy of Vibrio, Beneckea and Photobacterium: abolition of the genus Beneckea. Curr. Microbiol. 4:127-132.

6. Baumann, P., L. Baumann, and M. Mandel. 1971. Taxonomy of marine bacteria: the genus Beneckea. J. Bacteriol. 107:268-294.

7. Baumann, P., L. Baumann, M. Mandel, and R. D. Allen. 1971. Taxonomy of marine bacteria: Beneckea nigrapulchrituda sp. n. J. Bacteriol. 108:1380-1383.

8. Blake, P. A., M. H. Merson, R. E. Weaver, D. G. Hollis, and P. C. Heublein. 1979. Disease caused by a marine vibrio. Clinical characteristics and epidemiology. $\mathrm{N}$. Engl. J. Med. 300:1-5.

9. Blazevic, D. J., and G. M. Ederer. 1975. Principles of biochemical tests in diagnostic microbiology. John Wiley \& Sons, Inc., New York.

10. Breed, R. S., E. G. Murray, and N. R. Smith. 1957. Bergey's manual of determinative bacteriology, 7 th ed. The Williams \& Wilkins Co., Baltimore.

11. Citarella, R. V., and R. R. Colwell. 1970. Polyphasic taxonomy of the genus Vibrio: polynucleotide sequence relationships among selected Vibrio species. J. Bacteriol. 104:434-442.

12. Clark, W. A., and A. G. Steigerwalt. 1977. Deoxyribonucleic acid reassociation experiments with a halophilic, lactose-fermenting Vibrio isolated from blood cultures. Int. J. Syst. Bacteriol. 27:194-199.

13. Colwell, R. R. 1970. Polyphasic taxonomy of the genus Vibrio: numerical taxonomy of Vibrio cholerae, Vibrio parahaemolyticus, and related Vibrio species. J. Bacteriol. 104:410-433.

14. Colwell, R. R. 1974. Occurrence and biology of Vibrio 
parahaemolyticus, p. 230-240. In D. Schlessinger (ed.), Microbiology-1974. American Society for Microbiology, Washington, D.C.

15. Colwell, R. R., and R. Y. Morita. 1964. Reisolation and emendation of description of Vibrio marinus (Russel) Ford. J. Bacteriol. 88:831-837.

16. Colwell, R. R., R. J. Seidler, J. Kaper, S. W. Joseph, S. Garges, H. Lockman, D. Maneval, H. Bradford, N. Roberts, E. Remmers, I. Huq, and A. Huq. 1981. Occurrence of Vibrio cholerae serotype 0-1 in Maryland and Louisiana estuaries. Appl. Environ. Microbiol. 41:555558.

17. Colwell, R. R., and W. J. Wiebe. 1970. "Core" characteristics for use in classifying aerobic, heterotrophic bacteria by numerical taxonomy. Bull. Ga. Acad. Sci. 18:165-185.

18. Cowan, S. T. 1974. Cowan and Steel's manual for the identification of medical bacteria. Cambridge University Press, Cambridge.

19. Crosa, J. H., L. L. Hodges, and M. H. Schiewe. 1980. Curing of a plasmid is correlated with an attenuation of virulence in the marine fish pathogen Vibrio anguillarum. Infect. Immun. 27:879-902.

20. Crosa, J. H., M. H. Schiewe, and S. Falkow. 1977. Evidence for plasmid contribution to the virulence of the fish pathogen Vibrio anguillarum. Infect. Immun. 18:509_ 513.

21. Davey, R. B., and J. Pittard. 1975. Potential for in vivo acquisition of $\mathrm{R}$ plasmids by one strain of Vibrio cholerae biotype El Tor. Antimicrob. Agents Chemother. 8:111116.

22. Davis, B. R., G. R. Fanning, J. M. Madden, A. G. Steigerwalt, H. B. Bradford, Jr., H. L. Smith, Jr., and D. J. Brenner. 1981. Characterization of biochemically atypical Vibrio cholerae strains and designation of a new pathogenic species, Vibrio mimicus. J. Clin. Microbiol. 14:631-639.

23. Dubos, R. J. 1928. The decomposition of cellulose by aerobic bacteria. J. Bacteriol. 15:223-234.

24. Ducklow, H. W., H. M. Tarraza, Jr., and R. Mitchell. 1980. Experimental pathogenicity of Vibrio parahaemolyticus for the schistosome-bearing snail Biomphalaria glabrata. Can. J. Microbiol. 26:503-506.

25. Evelyn, T. P. T. 1971. First records of vibriosis in Pacific salmon cultured in Canada, and taxonomic status of the responsible bacterium, Vibrio anguillarum. J. Fish. Res. Board. Can. 28:517-525.

26. Ewing, W. H., and R. Hugh. 1974. Aeromonas, p. 230 237. In E. H. Lennette, E. H. Spaulding, and J. P. Truant (ed.), Manual of clinical microbiology. American Society for Microbiology, Washington, D.C.

27. Falcao, D. P., L. R. Trabulsi, F. W. Hickman, and J. J. Farmer III. 1975. Unusual Enterobacteriaceae: lactosepositive Salmonella typhimurium which is endemic in Sao Paulo, Brazil. J. Clin. Microbiol. 2:349-353.

28. Farmer, J. J., III. 1979. Vibrio ("Beneckea") vulnificus, the bacterium associated with sepsis, septicemia, and the sea. Lancet it:903.

29. Feeley, J. C., and A. Balows. 1974. Vibrio, p. 238-245. In E. H. Lennette, E. H. Spaulding, and J. R. Truant (ed.), Manual of clinical microbiology. American Society for Microbiology, Washington, D.C.

30. Finkelstein, R. A. 1973. Cholera. Crit. Rev. Microbiol. 2:553-623.

31. Fujino, T., R. Sakazaki, and K. Tamura. 1974. Designation of the type strain of Vibrio parahaemolyticus and description of 200 strains of the species. Int. J. Syst. Bacteriol. 24:447-449.

32. Fujino, T., R. Sakazaki, and K. Tamura. 1974. The proposed type strain of Vibrio parahaemolyticus, p. 159 161. In T. Fujino, G. Sakaguchi, R. Sakazaki, and Y. Takeda (ed.), International Symposium on V. parahae molyticus. Saiken Publishing Co., Tokyo.

33. Gilmour, A. 1977. Characteristics of marine vibrios isolated from fish farm tanks. Aquaculture 11:51-62.
34. Golten, C., and W. A. Schefiers. 1975. Marine vibrios isolated from water along the Dutch coast. Neth. J. Sea Res. 9:351-364.

35. Gordon, R. E. 1967. The taxonomy of soil bacteria, p. 293-321. In T. R. G. Gray and D. Parkinson (ed.), The ecology of soil bacteria. University Press, Liverpool.

36. Harrell, L. W., A. J. Novotny, M. H. Schiewe, and H. O. Hodgins. 1976. Isolation and description of two vibrios pathogenic to Pacific salmon in Puget Sound, Washington. Fish Bull. (U.S.) 74:447-449.

37. Harwood, C. S. 1978. Beneckea gazogenes sp. nov., a red, facultatively anaerobic, marine bacterium. Curr. Microbiol. 1:233-238.

38. Hastein, T., and J. E. Smith. 1977. A study of Vibrio anguillarum from farmed and wild fish using principal components analysis. J. Fish Biol. 11:69-75.

39. Heibert, B. 1936. The biochemical reaction of vibrios. J. Hyg. 36:114-117.

40. Hendrie, M. S., W. Hodgkiss, and J. M. Shewan. 1970. The identification, taxonomy and classification of luminous bacteria. J. Gen. Microbiol. 64:151-169.

41. Hendrie, M. S., W. Hodgkiss, and J. M. Shewan. 1971. Proposal that the species Vibrio anguillarum Bergman 1909, Vibrio piscium David 1927, and Vibrio ichthyodermis (Wells and Zobell) Shewan, Hobbs, and Hodgkiss 1960 be combined as a single species, Vibrio anguillarum. Int. J. Syst. Bacteriol. 21:64-68.

42. Hickman, F. W., J. J. Farmer, D. G. Hollis, G. R. Fanning, A. G. Steigerwalt, R. E. Weaver, and D. J. Brenner. 1982. Identification of Vibrio hollisae sp. nov. from patients with diarrhea. J. Clin. Microbiol. 15:395-401.

43. Hollis, D. G., R. E. Weaver, C. N. Baker, and C. Thornsberry. 1976. Halophilic Vibrio species isolated from blood cultures. J. Clin. Microbiol. 3:425-431.

44. Hsu, S. C., and J. L. Lockwood. 1975. Powdered chitin agar as a selective medium for enumeration of actinomycetes in water and soil. Appl. Microbiol. 29:422-426.

45. Hucker, G. J., and H. J. Conn. 1923. Methods of Gram staining. Technical Bulletin 93. New York State Agriculture Experiment Station, Ithaca.

46. Hugh, R., and G. L. Gilardi. 1974. Pseudomonas, p. 250-269. In E. H. Lennette, E. H. Spaulding, and J. P. Truant (ed.), Manual of clinical microbiology. American Society for Microbiology, Washington, D.C.

47. Hugh, R., and E. Leifson. 1953. The taxonomic significance of fermentative versus oxidative metabolism of carbohydrates by various gram-negative bacteria. J. Bacteriol. 66:24-26.

48. Hugh, R., and R. Sakazaki. 1975. Minutes of the meeting of the Subcommittee on the Taxonomy of Vibrios, 3 September 1974. Int. J. Syst. Bacteriol. 25:389-391.

49. Huq, M. I., A. K. M. J. Alam, D. J. Brenner, and G. K. Morris. 1980. Isolation of Vibrio-like group, EF-6, from patients with diarrhea. J. Clin. Microbiol. 11:621-624.

50. Huq, M. I., D. Huber, and G. Kibryia. 1979. Isolation of urease producing Vibrio parahaemolyticus strains from cases of gastroenteritis. Indian J. Med. Res. 70:540-553.

51. Jegathesan, M., and T. Paramasivam. 1976. Hydrogen sulfide production as an aid to the identification of Vibrio parahaemolyticus. Southeast Asian J. Trop. Med. Public Health 7:377-379.

52. Johnson, E. M., J. A. Wohlhieter, B. P. Placek, R. B. Sleet, and L. S. Baron. 1976. Plasmid-determined ability of a Salmonella tennessee strain to ferment lactose and sucrose. J. Bacteriol. 125:385-386.

53. Kampelmacher, E. H. 1977 . The spread and significance of salmonellae in surface waters in the Netherlands, $p$. 148-158. In A. W. Hoadley and D. J. Dutka (ed.), Bacterial indicators/health hazards associated with water. American Society for Testing and Materials, Philadelphia.

54. Kaper, J. B., S. L. Moseley, and S. Falkow. 1981. Molecular characterization of environmental and nontoxigenic Vibrio cholerae. Infect. Immun. 32:661-667.

55. Kiehn, E. D., and R. E. Pacha. 1969. Characterization 
and relatedness of marine vibrios pathogenic to fish: deoxyribonucleic acid homology and base composition. J. Bacteriol. 100:1248-1255.

56. King, E. O., M. K. Ward, and D. E. Raney. 1954. Two simple media for the demonstration of pyocyanin and fluorescein. J. Lab. Clin. Med. 44:301-307.

57. Kovacs, N. 1928. Eine vereinfachte Methode zum Nachweis der Indolbildung durch Bakterien. Z. Immunitaetsforsch. Exp. Ther. 55:311.

58. Kovacs, N. 1956. Identification of Pseudomonas pyocyanea by the oxidase reaction. Nature (London) 178:703.

59. Lam, S., and M. Yeo. 1980. Urease-positive Vibrio parahaemolyticus strain. J. Clin. Microbiol. 12:57-59.

60. Lee, J. V., T. J. Donovan, and A. L. Furniss. 1978. Characterization, taxonomy, and emended description of Vibrio metschnikovii. Int. J. Syst. Bacteriol. 28:99-111.

61. Lee, J. V., D. M. Glbeon, and J. M. Shewan. 1977. A numerical taxonomic study of some Pseudomonas-like marine bacteria. J. Gen. Microbiol. 98:439-451.

62. Lee, J. V., P. Shread, A. L. Furniss, and T. Bryant. 1981. Taxonomy and description of Vibrio fluvialis sp. nov. (synonym, group F vibrios, group EF-6). J. Appl. Bacteriol. 50:73-94.

63. Leifson, E. 1963. Determination of carbohydrate metabolism of marine bacteria. J. Bacteriol. 85:1183-1184.

64. Levin, M. A., R. E. Wolke, and V. J. Cabelli. 1972. Vibrio anguillarum as a cause of disease in winter flounder (Pseudopleuronectes americanus). Can. J. Microbiol. 18:1585-1592.

65. Love, M., D. Teebken-Fisher, J. E. Hose, J. J. Farmer, F. W. Hickman, and G. R. Fanning. 1981. Vibrio damsela, a marine bacterium, causes skin ulcers on the damselfish Chromis punctippinis. Science 214:11391140.

66. Lovelace, T. E., and R. R. Colwell. 1968. A multipoint inoculator for petri dishes. Appl. Microbiol. 16:944-945.

67. Moeller, V. 1955. Simplified tests for some amino acid decarboxylases and for the arginine dihydrolase system. Acta Pathol. Microbiol. Scand. 36:158-172.

68. Nybelin, O. 1935. Untersuchungen uber den bei Fischen Krankheitserregenden Spaltpilz Vibrio anguillarum. Medd. Untersokn. Anst. Sotvattensfisk. Stockholm 8:362.

69. Olsen, R. H., K. Carey, and D. Lorenz. 1975. Plasmid gene expression and possible hybridization between Vibrio cholerae and Escherichia coli, p. 134-143. In Proceedings of the 11 th Joint Conference on Cholera. National Institutes of Health, Bethesda, Md.

70. Pacha, R. E., and E. D. Kiehn. 1969. Characterization and relatedness of marine vibrios pathogenic to fish: physiology, serology, and epidemiology. J. Bacteriol. 100:1242-1247.

71. Popof, M., and M. Veron. 1976. A taxonomic study of the Aeromonas hydrophila-Aeromonas punctata group. J. Gen. Microbiol. 94:11-22.

72. Rahal, K., G. Gerbaud, and D. H. Bauanchaud. 1978. Stability of $\mathbf{R}$ plasmids belonging to different incompatability groups in Vibrio cholerae "El Tor." Ann. Microbiol. (Paris) 129A:409-414.

73. Reichelt, J. L., and P. Baumann. 1973. Taxonomy of the marine, luminous bacteria. Arch. Mikrobiol. 94:282-330.

74. Reichelt, J. L., P. Baumann, and L. Baumann. 1976. Study of genetic relationships among marine species of the genera Beneckea and Photobacterium by means of in vitro DNA/DNA hybridization. Arch. Microbiol. 110:101-120.

75. Sakazaki, R. 1968 . Proposal of Vibrio alginolyticus for the biotype 2 of Vibrio parahaemolyticus. Jpn. J. Med. Sci. Biol. 21:359-362.

76. Sakmaki, R., O. C. Z. Gomez, and M. Sebald. 1967. Taxonomic studies of the so-called NAG vibrios. Jpn. J. Med. Sci. Biol. 20:265-280.

77. Sakazaki, R., S. Iwanami, and H. Fukumi. 1963. Studies on the enteropathogenic, facultatively halophilic bacteri- um, Vibrio parahaemolyticus. I. Morphological, cultural and biochemical properties and its taxonomical position. Jpn. J. Med. Sci. Biol. 16:161-188.

78. Sanyal, S. C., R. Saknaki, L. M. Prescott, and R. Sinha. 1973. Isolation of rapidly lactose-fermenting Vibrio cholerae strains. J. Med. Microbiol. 6:119-120.

79. Schiewe, M. H., and J. H. Crose. 1981. Molecular characterization of Vibrio anguillarum biotype 2. Can. J. Microbiol. 27:1011-1018.

80. Schiewe, M. H., J. H. Crosa, and E. J. Ordal. 1977. Deoxyribonucleic acid relationships among marine vibrios pathogenic to fish. Can. J. Microbiol. 23:954-958.

81. Schubert, R. H. W. 1974. Genus Aeromonas Kluyver and van Niel, p. 345-348. In R. E. Buchanan and N. E. Gibbons (ed.), Bergey's manual of determinative bacteriology, 8th ed. The Williams \& Wilkins Co., Baltimore.

82. Shewan, J. M., and M. Veron. 1974. Genus Vibrio Pacini, p. 340-345. In R. E. Buchanan and N. E. Gibbons (ed.), Bergey's manual of determinative bacteriology, 8th ed. The Williams \& Wilkins Co., Baltimore.

83. Sierra, G. 1957. A simple method for the detection of lipolytic activity of microorganisms and some observations on the influence of the contact between cells and fatty substrates. Antonie van Leeuwenhoek J. Microbiol. Serol. 23:15-22.

84. Smith, H. L., Jr., and K. Goodner. 1958. Detection of bacterial gelatinases by gelatin agar plate methods. J. Bacteriol. 76:662-665.

85. Smith, I. W. 1961. A disease of finnock due to Vibrio anguillarum. J. Gen. Microbiol. 24:247-252.

86. Smith, R. E., and N. P. Willett. 1968. Rapid plate method for screening hyaluronidase- and chondroitin sulfataseproducing microorganisms. Appl. Microbiol. 16:1434 1436.

87. Sneath, P. H. A. 1956. Cultural and biochemical characteristics of the genus Chromobacterium. J. Gen. Microbiol. 15:70-98.

88. Sneath, P. H. A. 1957. The application of computers to taxonomy. J. Gen. Microbiol. 17:201-226.

89. Sneath, P. H. A., and R. R. Solral. 1973. Numerical taxonomy: the principles and practice of numerical classification. W. H. Freeman, San Francisco.

90. Sneath, P. H. A., and M. Stevens. 1967. A divided petri dish for use with multipoint inoculators. J. Appl. Bacteriol. 30:495-497.

91. Sokal, R. E., and C. D. Michener. 1958. A statistical method for evaluating systematic relationships. Univ. Kans. Sci. Bull. 38:1409-1438.

92. Staley, T. E., and R. R. Colwell. 1973. Deoxyribonucleic acid reassociation among members of the genus Vibrio. Int. J. Syst. Bacteriol. 23:316-332.

93. Stephen, S., A. L. Var, I. Chandrashekara, and K. N. A. Røo. 1978. Characterization of Vibrio alginolyticus (Beneckea alginolytica) isolated from the fauna of Arabian sea. Indian J. Med. Res. 68:7-11.

94. Stevenson, I. L. 1967. Utilization of aromatic hydrocarbons by Arthrobacter spp. Can. J. Microbiol. 13:205211.

95. Stuart, C. A., E. Van Stratum, and R. Rustigian. 1945. Further studies on urease production by Proteus and related organisms. J. Bacteriol. 49:437-444.

96. Threlfall, E. J., B. Rowe, and I. Huq. 1980. Plasmidencoded multiple antibiotic resistance in Vibrio cholerae El Tor from Bangladesh. Lancet i:1247-1248.

97. Turova, T. P., and A. S. Antonov, 1977. Similarity between polynucleotide sequences of DNA from cholera and the so-called non-agglutinating vibrios. Zh. Mikrobiol. Epidemiol. Immunobiol. 54:47-49.

98. Twedt, R. M., P. L. Spaulding, and H. E. Hall. 1969. Morphological, cultural, biochemical, and serological comparisons of Japanese strains of Vibrio parahaemolyticus with related cultures isolated in the United States. J. Bacteriol. 98:511-518.

99. West, P. A., R. M. Daniel, C. J. Knowles, and J. V. Lee. 1978. Tetramethyl-p-phenylenediame (TMPD) oxidase 
activity and cytochrome distribution in the genus Vibrio. FEMS Microbiol. Lett. 4:339-342.

100. Yokota, T., and S. Kuwahara. 1977. Temperature-sensitive $\mathbf{R}$ plasmid obtained from naturally isolated drugresistant Vibrio cholerae (biotype El Tor). Antimicrob.
Agents Chemother. 11:13-20.

101. Zen-Yoyi, H., S. Sakai, T. Terayama, Y. Kudo, M. Benoki, and M. Nagasaki. 1965. Epidemiology, enteropathogenicity, and classification of Vibrio parahaemolyticus. J. Infect. Dis. 115:436-444. 